TRANSACTIONS OF THE

AMERICAN MATHEMATICAL SOCIETY

Volume 365, Number 12, December 2013, Pages 6569-6593

S 0002-9947(2013)05920-X

Article electronically published on June 3, 2013

\title{
QUASILINEAR EQUATIONS WITH SOURCE TERMS ON CARNOT GROUPS
}

\author{
NGUYEN CONG PHUC AND IGOR E. VERBITSKY
}

\begin{abstract}
In this paper we give necessary and sufficient conditions for the existence of solutions to quasilinear equations of Lane-Emden type with measure data on a Carnot group $\mathbb{G}$ of arbitrary step. The quasilinear part involves operators of the $p$-Laplacian type $\Delta_{\mathbb{G}, p}, 1<p<\infty$. These results are based on new a priori estimates of solutions in terms of nonlinear potentials of Th. Wolff's type. As a consequence, we characterize completely removable singularities, and we prove a Liouville type theorem for supersolutions of quasilinear equations with source terms which has been known only for equations involving the sub-Laplacian $(p=2)$ on the Heisenberg group.
\end{abstract}

\section{INTRODUCTION}

In this paper we study the solvability problem and pointwise estimates of solutions for a class of quasilinear Lane-Emden type equations with measure data on Carnot groups of arbitrary step. A complete characterization of removable singularities for the corresponding homogeneous equations as well as a Liouville type theorem for supersolutions will also be obtained as a consequence.

The basic setting of our study is a given Carnot group $\mathbb{G}$ of step $r \geq 1$, i.e., a connected and simply connected stratified nilpotent Lie group whose Lie algebra $\mathcal{G}$ admits a stratification $\mathcal{G}=V_{1} \oplus V_{2} \oplus \cdots \oplus V_{r}$ and is generated via commutations by its first (horizontal) layer $V_{1}$ (see Section 2). Given a basis $\left\{X_{j}\right\}_{j=1}^{m}$ of $V_{1}$, the associated $p$-Laplacian operator $\Delta_{\mathbb{G}, p}, 1<p<\infty$, is defined by

$$
\Delta_{\mathbb{G}, p} u=\sum_{i=1}^{m} X_{i}\left(|X u|^{p-2} X_{i} u\right),
$$

where

$$
X u=X_{1} u X_{1}+X_{2} u X_{2}+\cdots+X_{m} u X_{m},
$$

is the horizontal gradient of $u$ with length $|X u|=\left(\sum_{i=1}^{m}\left|X_{i} u\right|^{2}\right)^{1 / 2}$.

We study the following Lane-Emden type equation on a bounded open set $\Omega \subset \mathbb{G}$ :

$$
\left\{\begin{aligned}
-\Delta_{\mathbb{G}, p} u & =u^{q}+\omega \text { in } \Omega, \\
u & =0 \text { on } \partial \Omega
\end{aligned}\right.
$$

where $q>p-1>0$, and $\omega$ is a given nonnegative finite measure on $\Omega$. Our objective is to obtain necessary and sufficient conditions on the measure $\omega$ for the

Received by the editors December 18, 2011 and, in revised form, July 3, 2012.

2010 Mathematics Subject Classification. Primary 35H20; Secondary 35A01, 20F18.

The first author was supported in part by NSF Grant DMS-0901083.

The second author was supported in part by NSF Grant DMS-0901550. 
existence of solutions to (1.1) and to give a complete characterization of removable singularities for the corresponding homogeneous equation,

$$
-\Delta_{\mathbb{G}, p} u=u^{q} \quad \text { in } \Omega .
$$

Equations similar to (1.1) in the entire group $\mathbb{G}$ are also considered with applications to Liouville type theorems for the differential inequality

$$
-\Delta_{\mathbb{G}, p} u \geq u^{q} \quad \text { in } \mathbb{G} .
$$

Such problems have been studied in depth in our previous work PV1, [PV2] in the standard Euclidean setting; see also earlier work in [BP], $[\mathrm{AP}$, BV1], and BV2. However, in the setting of Carnot groups, the failure of the Besicovitch covering lemma (see [SW], $[\mathrm{KR}]$ ) and the lack of a perfect dyadic grid of cubes cause major difficulties. We observe that even in the setting of the Heisenberg group, the simplest model of a noncommutative Carnot group, Liouville type theorems for the differential inequality (1.3) are known only in the sub-Laplacian case, i.e., $p=2$ (see [GL, [BCC], PVe]).

A substantial part of our study of (1.1) is devoted to integral inequalities for both linear and nonlinear potential operators and their discrete analogues over "approximate" dyadic grids of cubes constructed in [SW] and [Chr] in the general setting of homogeneous spaces.

For each $\alpha>0$, the Bessel potential of a locally integrable function $f$ in this setting is defined by

$$
\mathbf{G}_{\alpha}(f)(x)=\mathbf{G}_{\alpha} * f(x)=\int_{\mathbb{G}} \mathbf{G}_{\alpha}\left(y^{-1} x\right) f(y) d y, \quad x \in \mathbb{G},
$$

where $\mathbf{G}_{\alpha}$ is the Bessel kernel of order $\alpha$ on $\mathbb{G}$ given by

$$
\mathbf{G}_{\alpha}(x)=\frac{1}{\Gamma(\alpha / 2)} \int_{0}^{\infty} t^{\alpha / 2-1} e^{-t} h(x, t) d t .
$$

In (1.4) $h(x, t)$ is the heat kernel associated with the sub-Laplacian $\Delta_{\mathbb{G}}=\Delta_{\mathbb{G}, 2}$ whose basic properties can be found in [Fol], VSC]. We also write

$$
\mathbf{G}_{\alpha}(f d \mu)(x)=\mathbf{G}_{\alpha} *(f d \mu)(x)=\int_{\mathbb{G}} \mathbf{G}_{\alpha}\left(y^{-1} x\right) f(y) d \mu(y), \quad x \in \mathbb{G},
$$

for each locally $\mu$-integrable function $f$.

When dealing with solutions on the entire group $\mathbb{G}$ and Liouville type theorems, we need to use another linear potential, the Riesz potential. For each $0<\alpha<M$ and $f \in L_{\mathrm{loc}}^{1}(\mathbb{G})$, it is defined by

$$
\mathbf{I}_{\alpha}(f)(x)=\mathbf{I}_{\alpha} * f(x)=\int_{\mathbb{G}} \frac{f(y)}{\left|y^{-1} x\right|^{M-\alpha}} d y, \quad x \in \mathbb{G},
$$

where $|\cdot|$ stands for the homogeneous norm on $\mathbb{G}$, and $M$ is the homogeneous dimension of $\mathbb{G}$ (see Section 2).

Associated with the kernel $\mathbf{G}_{\alpha}$ is the Bessel capacity $C_{\alpha, s}(\cdot), s>1$, defined by (see $[\mathrm{AH}]$, Sec. 2.6, in the Euclidean case)

$$
C_{\alpha, s}(E)=\inf \left\{\|f\|_{L^{s}(\mathbb{G})}^{s}: \mathbf{G}_{\alpha}(f) \geq 1 \text { on } E, f \in L^{s}(\mathbb{G}), f \geq 0\right\}
$$

for each compact set $E \subset \mathbb{G}$. Similarly, the Riesz capacity $\dot{C}_{\alpha, s}(\cdot), 0<\alpha<M$, $s>1$, is defined, for a compact set $E \subset \mathbb{G}$, by

$$
\dot{C}_{\alpha, s}(E)=\inf \left\{\|f\|_{L^{s}(\mathbb{G})}^{s}: \mathbf{I}_{\alpha}(f) \geq 1 \text { on } E, f \in L^{s}(\mathbb{G}), f \geq 0\right\} .
$$


These capacities will play an essential role in our characterizations of the existence of solutions and removable singularities, as well as Liouville type theorems for the Lane-Emden type equation. We will also need the following dual definition of these capacities (see [Lu, Theorem 2.10]; $\mathrm{AH}$, Theorem 2.2.7] in the Euclidean case):

$$
C_{\alpha, s}(E)=\sup _{\mu \in \mathcal{M}^{+}(E), \mu \neq 0}\left(\frac{\mu(E)}{\left\|\mathbf{G}_{\alpha} * \mu\right\|_{L^{\frac{s}{s-1}}(\mathbb{G})}}\right)^{s}
$$

and similarly,

$$
\dot{C}_{\alpha, s}(E)=\sup _{\mu \in \mathcal{M}^{+}(E), \mu \neq 0}\left(\frac{\mu(E)}{\left\|\mathbf{I}_{\alpha} * \mu\right\|_{L^{\frac{s}{s-1}}(\mathbb{G})}}\right)^{s},
$$

where $\mathcal{M}^{+}(E)$ denotes the set of all nonnegative measures supported on $E$.

The nonlinear potential we use below is the (truncated) Wolff's potential $\mathbf{W}_{\alpha, p}^{R}$ originally introduced in [HW]. In our setting, for $\alpha>0, p>1$, and $0<R \leq \infty$, it is defined for each nonnegative measure $\mu$ on $\mathbb{G}$ by

$$
\mathbf{W}_{\alpha, p}^{R} \mu(x)=\int_{0}^{R}\left[\frac{\mu\left(B_{t}(x)\right)}{t^{M-\alpha p}}\right]^{\frac{1}{p-1}} \frac{d t}{t}, \quad x \in \mathbb{G},
$$

where $B_{t}(x)$ is the Carnot-Carathéodory ball centered at $x$ of radius $t$ (see Section 2).

For our purpose we introduce the following notion of solutions for $p$-Laplace equations with general measure as data. This will serve as an efficient substitution for the notion of renormalized solutions introduced in DMOP in the Euclidean setting.

Definition 1.1. For a nonnegative finite measure $\mu$ on $\Omega$, we say that $u$ is a solution to

$$
\left\{\begin{array}{rll}
-\Delta_{\mathbb{G}, p} u & =\mu & \text { in } \Omega \\
u & =0 & \text { on } \partial \Omega
\end{array}\right.
$$

in the potential theoretic sense if $u$ is $p$-superharmonic in $\Omega, \min \{u, k\} \in S_{0}^{1, p}(\Omega)$ for every $k>0, u$ satisfies a pointwise bound

$$
u(x) \leq A \mathbf{W}_{1, p}^{2 \operatorname{diam}(\Omega)} \mu(x), \quad \forall x \in \Omega,
$$

and for every $\varphi \in C_{0}^{\infty}(\Omega)$ one has

$$
\int_{\Omega}|X u|^{p-2} X u \cdot X \varphi d x=\int_{\Omega} \varphi d \mu .
$$

From this definition we see right away that potential theoretic solutions to (1.7) are also distributional solutions. However, the converse is not necessarily true as easily seen by a simple example (see [Kil]). The existence of potential theoretic solutions to (1.7) will be obtained in Corollary 4.2, whereas their uniqueness is unknown even in the Euclidean setting.

In Definition 1.1 the notation $S_{0}^{1, p}(\Omega)$ stands for the completion of $C_{0}^{\infty}(\Omega)$ under the norm of the horizontal Sobolev space $S^{1, p}(\Omega)$ (see Section 3 ), and in (1.8), $A$ is a universal constant independent of $x, u, \mu$, and $\Omega$. For the notion of $p$-superharmonic functions on Carnot groups, see Section 3. We are now ready to state the first result of the paper. 
Theorem 1.2. Let $p>1, q>p-1$, and $R=\operatorname{diam}(\Omega)$. Suppose that $\omega$ is a nonnegative finite measure on $\Omega$ such that $\operatorname{supp}(\omega) \Subset \Omega$. If the equation

$$
\left\{\begin{aligned}
-\Delta_{\mathbb{G}, p} u & =u^{q}+\omega \text { in } \Omega, \\
u & =0 \text { on } \partial \Omega
\end{aligned}\right.
$$

has a nonnegative $p$-superharmonic distributional solution $u \in L^{q}(\Omega)$, then there exists a constant $C>0$ such that statements (i)-(v) below hold true.

(i) The inequality

$$
\int_{\mathbb{G}} \mathbf{G}_{p}(f)^{\frac{q}{q-p+1}} d \omega \leq C \int_{\mathbb{G}} f^{\frac{q}{q-p+1}} d x
$$

holds for all $f \in L^{\frac{q}{q-p+1}}, f \geq 0$.

(ii) For every compact set $E \subset \Omega$,

$$
\omega(E) \leq C C_{p, \frac{q}{q-p+1}}(E) .
$$

(iii) The inequality

$$
\int_{\mathbb{G}}\left[\mathbf{W}_{1, p}^{2 R}(g d \omega)(x)\right]^{q} d x \leq C \int_{\mathbb{G}} g^{\frac{q}{p-1}} d \omega
$$

holds for all $g \in L^{\frac{q}{p-1}}(d \omega), g \geq 0$.

(iv) The inequality

$$
\int_{B}\left[\mathbf{W}_{1, p}^{2 R} \omega_{B}(x)\right]^{q} d x \leq C \omega(B)
$$

holds for all Carnot-Carathéodory balls $B \subset \mathbb{G}$.

(v) For all $x \in \Omega$,

$$
\mathbf{W}_{1, p}^{2 R}\left[\left(\mathbf{W}_{1, p}^{2 R} \omega\right)^{q}\right](x) \leq C \mathbf{W}_{1, p}^{2 R} \omega(x) .
$$

Conversely, there exists a constant $C_{0}=C_{0}(M, p, q)>0$ such that the following holds. Suppose that $\omega$ is a nonnegative finite measure for which one of the statements (i)-(v) holds with $C \leq C_{0}$, then equation (1.9) has a nonnegative potential theoretic solution $u \in L^{q}(\Omega)$. Moreover, u satisfies the following pointwise estimate

$$
u \leq \kappa \mathbf{W}_{1, p}^{2 R} \omega .
$$

Our second result characterizes removable singularities of solutions to homogeneous equations, which is in fact a consequence of Theorem 1.2 .

Theorem 1.3. Let $q>p-1>0$, and let $E$ be a compact subset of $\Omega$. Then any solution $u$ to the problem

$$
\left\{\begin{array}{c}
u \text { is } p \text {-superharmonic in } \Omega \backslash E, \\
u \in L_{\text {loc }}^{q}(\Omega \backslash E), \quad u \geq 0, \\
-\Delta_{\mathbb{G}, p} u=u^{q} \quad \text { in } \quad \mathcal{D}^{\prime}(\Omega \backslash E)
\end{array}\right.
$$

is also a solution to a similar problem with $\Omega$ in place of $\Omega \backslash E$ if and only if

$$
C_{p, \frac{q}{q-p+1}}(E)=0 .
$$

The proofs of Theorems 1.2 and 1.3 will be given at the end of Section 4 . In case the bounded domain $\Omega$ in Theorem 1.2 is replaced by the whole group $\mathbb{G}$, then Riesz potentials and the corresponding Riesz capacity must be used, and we have the following result. 
Theorem 1.4. Let $1<p<M, q>p-1$, and let $\omega$ be a nonnegative locally finite measure on $\mathbb{G}$. If the equation

$$
\left\{\begin{aligned}
-\Delta_{\mathbb{G}, p} u & =u^{q}+\omega \text { in } \mathbb{G} \\
\inf _{\mathbb{G}} u & =0
\end{aligned}\right.
$$

has a nonnegative p-superharmonic distributional solution $u \in L_{\mathrm{loc}}^{q}(\mathbb{G})$, then there exists a constant $C>0$ such that statements (i)-(vi) below hold true.

(i) For every compact set $E \subset \mathbb{G}$,

$$
\int_{E} u^{q} d x \leq C \dot{C}_{p, \frac{q}{q-p+1}}(E) .
$$

(ii) The inequality

$$
\int_{\mathbb{G}} \mathbf{I}_{p}(f)^{\frac{q}{q-p+1}} d \omega \leq C \int_{\mathbb{G}} f^{\frac{q}{q-p+1}} d x
$$

holds for all $f \in L^{\frac{q}{q-p+1}}, f \geq 0$.

(iii) For every compact set $E \subset \mathbb{G}$,

$$
\omega(E) \leq C \dot{C}_{p, \frac{q}{q-p+1}}(E) .
$$

(iv) The inequality

$$
\int_{\mathbb{G}}\left[\mathbf{W}_{1, p}^{\infty}(g d \omega)(x)\right]^{q} d x \leq C \int_{\mathbb{G}} g^{\frac{q}{p-1}} d \omega
$$

holds for all $g \in L^{\frac{q}{p-1}}(d \omega), g \geq 0$.

(v) The inequality

$$
\int_{B}\left[\mathbf{W}_{1, p}^{\infty} \omega_{B}(x)\right]^{q} d x \leq C \omega(B)
$$

holds for all Carnot-Carathéodory balls $B \subset \mathbb{G}$.

(vi) For all $x \in \Omega$,

$$
\mathbf{W}_{1, p}^{\infty}\left[\left(\mathbf{W}_{1, p}^{\infty} \omega\right)^{q}\right](x) \leq C \mathbf{W}_{1, p}^{\infty} \omega(x) .
$$

Conversely, there exists a constant $C_{0}=C_{0}(M, p, q)>0$ such that if any one of the statements (ii)-(vi) holds with $C \leq C_{0}$, then equation (1.15) has a nonnegative p-superharmonic solution $u \in L_{\mathrm{loc}}^{q}(\mathbb{G})$. Moreover, u satisfies the following pointwise two-sided estimate

$$
\kappa_{1} \mathbf{W}_{1, p}^{\infty} \omega \leq u \leq \kappa_{2} \mathbf{W}_{1, p}^{\infty} \omega .
$$

Theorem 1.4 yields the following Liouville type theorem. We observe that for $p \neq 2$ this Liouville type theorem is new even in the Heisenberg group. For $p=2$, as mentioned earlier, such a result was obtained in GL, BCC], and [PVe in the setting of the Heisenberg group. However, the approach of using test functions and integration by parts in these papers does not seem to work in the general setting of Carnot groups of arbitrary step.

Corollary 1.5. If $q \leq \frac{M(p-1)}{M-p}$, then the inequality $-\Delta_{\mathbb{G}, p} u \geq u^{q}$ admits no nontrivial nonnegative $p$-superharmonic distributional solutions in $\mathbb{G}$.

The proofs of Theorem 1.4 and Corollary 1.5 will be given in Section 5 . 


\section{Preliminaries on Carnot groups}

Let $\mathbb{G}$ be a Lie group, i.e., a differentiable manifold endowed with a group structure such that the map $\mathbb{G} \times \mathbb{G} \rightarrow \mathbb{G}$ defined by $(x, y) \mapsto x y^{-1}$ is $C^{\infty}$. Here $y^{-1}$ is the inverse of $y$ and $x y^{-1}$ denotes the group multiplication of $x$ by $y^{-1}$. We will denote by

$$
L_{x_{0}}(x)=x_{0} x, \quad R_{x_{0}}(x)=x x_{0},
$$

respectively, the left- and right-translations on $\mathbb{G}$. A vector field $X$ on $\mathbb{G}$ is called left-invariant if for each $x_{0} \in \mathbb{G}$,

$$
d L_{x_{0}}(X(x))=X\left(x_{0} x\right)
$$

for all $x \in \mathbb{G}$, i.e., $d L_{x_{0}} \circ X=X \circ L_{x_{0}}$. Here $d L_{x_{0}}$ is the differential of $L_{x_{0}}$. Under the Lie bracket operation on vector fields, the set of left-invariant vector fields on $\mathbb{G}$ forms a Lie algebra called the Lie algebra of $\mathbb{G}$ and is denoted by $\mathcal{G}$. Note that we can identify $\mathcal{G}$ with the tangent space $\mathbb{G}_{e}$ to $\mathbb{G}$ at the identity $e \in \mathbb{G}$ via the isomorphism $\alpha: \mathcal{G} \rightarrow \mathbb{G}_{e}$ defined by $\alpha(X)=X(e)$ and thus $\operatorname{dim} \mathcal{G}=\operatorname{dim} \mathbb{G}=N$, the topological dimension of $\mathbb{G}$.

A Carnot group $\mathbb{G}$ of step $r$ is a connected and simply connected Lie group whose Lie algebra $\mathcal{G}$ admits a nilpotent stratification of step $r$, i.e., $\mathcal{G}=V_{1} \oplus V_{2} \oplus \cdots \oplus V_{r}$ with $\left[V_{1}, V_{i}\right]=V_{i+1}$ for $i=1, \ldots, r-1, V_{r} \neq\{0\}$ and $\left[V_{1}, V_{r}\right]=0$, where $[\cdot, \cdot]$ denotes the Lie bracket.

Let $\left\{X_{j}\right\}_{j=1}^{m}$ be a basis for the first layer $V_{1}$ (also called the horizontal layer) of $\mathcal{G}$. Then for $2 \leq i \leq r$, we can choose a basis $\left\{X_{i j}\right\}, 1 \leq j \leq \operatorname{dim}\left(V_{i}\right)$, for $V_{i}$ consisting of commutators of length $i$. In particular, $X_{1 j}=X_{j}$ for $j=1, \ldots, m$, and $m=\operatorname{dim}\left(V_{1}\right)$. We then define an inner product $\langle\cdot, \cdot\rangle$ on $\mathcal{G}$ by declaring the $X_{i j}$ 's to be orthonormal. Since $\mathbb{G}$ is connected and simply connected, the exponential map exp is a global diffeomorphism from $\mathcal{G}$ onto $\mathbb{G}$ (see VSC], Va]). Thus for each $x \in \mathbb{G}$, there is a unique $\hat{x}=\left(x_{i j}\right) \in \mathbb{R}^{N}, 1 \leq i \leq r, 1 \leq j \leq \operatorname{dim}\left(V_{i}\right)$, and $N=\sum_{i=1}^{r} \operatorname{dim}\left(V_{i}\right)$, the topological dimension of $\mathbb{G}$, such that

$$
x=\exp \left(\sum x_{i j} X_{i j}\right) .
$$

Thus the maps $\phi_{i j}: \mathbb{G} \rightarrow \mathbb{R}, 1 \leq i \leq r, 1 \leq j \leq \operatorname{dim}\left(V_{i}\right)$, defined by

$$
\phi_{i j}(x)=x_{i j} \quad \text { for } x=\exp \left(\sum x_{i j} X_{i j}\right),
$$

form a system of global coordinates on $\mathbb{G}$ which are called the exponential coordinates. Henceforth, we will always use these coordinates and simply write

$$
x=\left(\phi_{i j}(x)\right)=\left(x_{i j}\right) \quad \text { for } x=\exp \left(\sum x_{i j} X_{i j}\right) .
$$

Such an identification of $\mathbb{G}$ with its Lie algebra is justified by the Baker-CambellHausdorff formula (see, e.g., $\mathrm{Va}$ )

$$
\exp \left(\sum x_{i j} X_{i j}\right) \exp \left(\sum y_{i j} X_{i j}\right)=\exp \left[H\left(\sum x_{i j} X_{i j}, \sum y_{i j} X_{i j}\right)\right]
$$

where $H(X, Y)=X+Y+\frac{1}{2}[X, Y]+\cdots$ with the dots indicating a finite linear combination of terms containing commutators of order two and higher. If we define a group law $*$ on $\mathcal{G}$ by

$$
X * Y=H(X, Y)
$$


then the group $\mathbb{G}$ can be identified with $(\mathcal{G}, *)$ via the exponential coordinates. Note that from the Baker-Cambell-Hausdorff formula we have

$$
\phi_{i j}\left(x_{0} x\right)=\phi_{i j}\left(x_{0}\right)+\phi_{i j}(x)+P_{i j}\left(x_{0}, x\right),
$$

where $P_{i j}\left(x_{0}, x\right)$ depends only on the coordinates $\phi_{k l}\left(x_{0}\right)$ and $\phi_{k l}(x)$ with $k<i$. Thus the determinant of $d L_{x_{0}}$ is equal to 1 , and the same properties hold for the right translation $R_{x_{0}}$ and its differential $d R_{x_{0}}$ as well. It follows that Lebesgue measure on $\mathcal{G}$ is lifted via the exponential mapping exp to a bi-invariant Haar measure on $\mathbb{G}$, which we will denote by $d x$.

For a given function $f: \mathbb{G} \rightarrow \mathbb{R}$, the action of $X \in \mathcal{G}$ on $f$ is specified by the equation

$$
X f(x)=\lim _{t \rightarrow 0} \frac{f(x \exp (t X))-f(x)}{t}=\left.\frac{d}{d t} f(x \exp (t X))\right|_{t=0} .
$$

For $t>0$, we define the dilation $\delta_{t}: \mathbb{G} \rightarrow \mathbb{G}$ by

$$
\delta_{t}(x)=\left(t^{i} \phi_{i j}(x)\right)
$$

whose Jacobian determinant is everywhere equal to $t^{M}$, where

$$
M=\sum_{i=1}^{r} i \operatorname{dim}\left(V_{i}\right)
$$

is the homogeneous dimension of $G$. A homogeneous norm $|\cdot|$ on $\mathbb{G}$ is defined by

$$
|x|=\left(\sum\left|\phi_{i j}(x)\right|^{2 r ! / i}\right)^{1 / 2 r !}
$$

which obviously satisfies $\left|\delta_{t}(x)\right|=t|x|$ and $\left|x^{-1}\right|=|x|$. This homogeneous norm generates a quasimetric $\rho(x, y)=\left|x^{-1} y\right|$ equivalent to the Carnot-Carathéodory metric $d_{c c}$ on $\mathbb{G}$ (see $\mathrm{NSW}$, [VSC]). Here

$$
d_{c c}(x, y)=\inf _{\gamma} \int_{a}^{b} \sqrt{\langle\dot{\gamma}(t), \dot{\gamma}(t)\rangle} d t
$$

where the infimum is taken over all curves $\gamma:[a, b] \rightarrow \mathbb{G}$ such that $\gamma(a)=x$, $\gamma(b)=y$ and $\dot{\gamma}(t) \in V_{1}$ for all $t$. Such a curve is called a horizontal curve connecting $x, y \in \mathbb{G}$. By Chow-Rashevsky's accessibility theorem (see [Cho, Ra]), any two points $x, y \in \mathbb{G}$ can be joined by a horizontal curve of finite length and hence $d_{c c}$ is a left-invariant metric on $\mathbb{G}$. We will denote by

$$
B_{R}(x)=\left\{y \in \mathbb{G}: d_{c c}(x, y)<R\right\}
$$

the Carnot-Carathéodory metric ball centered at $x$ with radius $R$. Note that there is $c=c(\mathbb{G})$ such that

$$
\left|B_{R}(x)\right|=c R^{M},
$$

where for a Borel set $E \subset \mathbb{G}$ we write $|E|$ for $\int_{E} d x$. Moreover, by homogeneity and left-invariance we have

$$
\left|\delta_{t}(E)\right|=t^{M}|E|, \quad d\left(\delta_{t}(x)\right)=t^{M} d x
$$

and for $x, x^{\prime}, y \in \mathbb{G}$,

$$
d_{c c}\left(y x, y x^{\prime}\right)=d_{c c}\left(x, x^{\prime}\right), \quad B_{R}(x)=x B_{R}(e) .
$$




\section{3. $p$-SUPERHARMONIC FUnCTIONS ON CARNOT GROUPS}

Let $p>1$, and let $\Omega$ be an open set in $\mathbb{G}$. Recall from the previous section that $X=\left(X_{1}, X_{2}, \ldots, X_{m}\right)=\left(X_{11}, X_{12}, \ldots, X_{1 m}\right)$ is an orthonormal basic for the first layer $V_{1}$ of $\mathcal{G}$. The horizontal Sobolev space $S^{1, p}(\Omega)$ associated with the system $X$ is defined by

$$
S^{1, p}(\Omega)=\left\{u \in L^{p}(\Omega): X_{i} u \in L^{p}(\Omega), i=1, \ldots, m\right\},
$$

where $X_{i} u$ is understood in the sense of distributions, i.e.,

$$
X_{i} u(\varphi)=-\int_{\Omega} u X_{i} \varphi d x
$$

for every $\varphi \in C_{0}^{\infty}(\Omega)$. It is a Banach space equipped with the norm

$$
\|u\|_{S^{1, p}(\Omega)}=\left(\int_{\Omega}\left(|u|^{p}+|X u|^{p}\right)\right)^{\frac{1}{p}}
$$

The corresponding local Sobolev space $S_{\text {loc }}^{1, p}(\Omega)$ is defined similarly, with $L_{\text {loc }}^{p}(\Omega)$ in place of $L^{p}(\Omega)$. We will denote by $S_{0}^{1, p}(\Omega)$ the completion of $C_{0}^{\infty}(\Omega)$ under the norm $\|\cdot\|_{S^{1, p}(\Omega)}$.

Recall that for a smooth function $u$ on $\mathbb{G}$, the $p$-Laplacian of $u$ is defined by

$$
\Delta_{\mathbb{G}, p} u=\sum_{i=1}^{m} X_{i}\left(|X u|^{p-2} X_{i} u\right),
$$

where $X u=X_{1} u X_{1}+X_{2} u X_{2}+\cdots+X_{m} u X_{m}$ is the horizontal gradient of $u$, and $|X u|^{2}=\sum_{i=1}^{m}\left|X_{i} u\right|^{2}$. A function $u \in S_{\mathrm{loc}}^{1, p}(\Omega)$ is said to be a weak solution to

$$
\Delta_{\mathbb{G}, p} u=0
$$

if

$$
\int_{\Omega}|X u|^{p-2} X u \cdot X \varphi d x=0
$$

for every $\varphi \in C_{0}^{\infty}(\Omega)$. Here $X u \cdot X \varphi=\sum_{i=1}^{m} X_{i} u X_{i} \varphi$. It is known that every weak solution to (3.1) has a continuous representative (see [CDG], [HH]), and such continuous solutions are called p-harmonic functions on $\Omega$. On the other hand, if $u \in S_{\mathrm{loc}}^{1, p}(\Omega)$ and

$$
\int_{\Omega}|X u|^{p-2} X u \cdot X \varphi d x \geq 0
$$

for every $\varphi \in C_{0}^{\infty}(\Omega), \varphi \geq 0$, then $u$ is called a supersolution to (3.1).

A lower semicontinuous function $u: \Omega \rightarrow(-\infty, \infty]$ is called $p$-superharmonic if $u$ is not identically infinite in each component of $\Omega$, and if for all open sets $D$ such that $\bar{D} \subset \Omega$, and all functions $v \in C(\bar{D}), p$-harmonic in $D$, it follows that $v \leq u$ on $\partial D$ implies $v \leq u$ in $D$.

The following fundamental connection between supersolutions to (3.1) and $p$ superharmonic functions can be found in [TW].

Proposition 3.1. Let $u \in S_{\mathrm{loc}}^{1, p}(\Omega)$ be a supersolution to (3.1). Let

$$
\underline{u}(x)=\operatorname{ess} \liminf _{y \rightarrow x} u(y) .
$$

Then $\underline{u}$ is p-superharmonic and $u=\underline{u}$ a.e. 
From this proposition it follows that we may assume all supersolutions to be lower semicontinuous. Therefore a function $u$ is a supersolution to (3.1) if and only if $u$ is $p$-superharmonic and belongs to $S_{\mathrm{loc}}^{1, p}(\Omega)$.

Note that a $p$-superharmonic function $u$ does not necessarily belong to $S_{\text {loc }}^{1, p}(\Omega)$, but its truncation $\min \{u, k\}$ does for every integer $k$. Using this we set

$$
X u=\lim _{k \rightarrow \infty} X[\min \{u, k\}],
$$

defined a.e. If either $u \in L^{\infty}(\Omega)$ or $u \in S_{\text {loc }}^{1,1}(\Omega)$, then $X u$ coincides with the regular distributional horizontal gradient of $u$. In general we have the following gradient estimate [TW] (see also [HKM] in the Euclidean case).

Proposition 3.2 ([TW]). Suppose $u$ is p-superharmonic in $\Omega$. Then $X u$ belongs to $L_{\mathrm{loc}}^{r}(\Omega)$, where $r<\frac{M(p-1)}{M-1}$.

From Proposition 3.2 and the dominated convergence theorem we have

$$
\int_{\Omega}|X u|^{p-2} X u \cdot X \varphi d x=\lim _{k \rightarrow \infty} \int_{\Omega}\left|X u_{k}\right|^{p-2} X u_{k} \cdot X \varphi d x \geq 0,
$$

whenever $\varphi \in C_{0}^{\infty}(\Omega)$ and $\varphi \geq 0$, where $u_{k}=\min \{u, k\}$. Thus the map

$$
\varphi \mapsto \int_{\Omega}|X u|^{p-2} X u \cdot X \varphi d x
$$

is a nonnegative distribution in $\Omega$ for a $p$-superharmonic function $u$. It follows that there is a positive (not necessarily finite) Radon measure denoted by $\mu[u]$ such that

$$
\int_{\Omega}|X u|^{p-2} X u \cdot X \varphi d x=\int_{\Omega} \varphi d \mu[u], \quad \forall \varphi \in C_{0}^{\infty}(\Omega),
$$

or in short we write

$$
-\Delta_{\mathbb{G}, p} u=\mu[u] \text { in } \Omega .
$$

The close relation between $p$-superharmonic functions and measures generated by them is established in the weak continuity theorem due to Trudinger and Wang TW.

Theorem 3.3 ([TW]). Suppose that $\left\{u_{n}\right\}$ is a sequence of nonnegative p-superharmonic functions in $\Omega$ that converges a.e. to a p-superharmonic function $u$. Then the sequence of corresponding measures $\left\{\mu\left[u_{n}\right]\right\}$ converges to $\mu[u]$ weakly, i.e.,

$$
\lim _{n \rightarrow \infty} \int_{\Omega} \varphi d \mu\left[u_{n}\right]=\int_{\Omega} \varphi d \mu[u]
$$

for all $\varphi \in C_{0}^{\infty}(\Omega)$.

The following pointwise estimates by means of Wolff's potentials were also proved in [TW] extending earlier results due to Kilpeläinen and Malý [KM2] to the subelliptic setting. They will play an essential role in this paper.

Theorem 3.4 ([TW]). Suppose $u \geq 0$ is a p-superharmonic function in $B_{3 r}(x)$. If $\mu=-\Delta_{\mathbb{G}, p} u$, then

$$
C_{1} \mathbf{W}_{1, p}^{r} \mu(x) \leq u(x) \leq C_{2} \inf _{B_{r}(x)} u+C_{3} \mathbf{W}_{1, p}^{2 r} \mu(x),
$$

where $C_{1}, C_{2}$ and $C_{3}$ are positive constants depending only on $M$ and $p$. Consequently, if $-\Delta_{\mathbb{G}, p} u=\mu$ on $\mathbb{G}$ and $\inf _{\mathbb{G}} u=0$, then

$$
C_{1} \mathbf{W}_{1, p}^{\infty} \mu(x) \leq u(x) \leq C_{3} \mathbf{W}_{1, p}^{\infty} \mu(x) .
$$




\section{LANE-EMden TyPE EQUATIONS AND RELATED INEQUALITIES}

In this section we fix a standard mollifier $\zeta$ on $\mathbb{G}$, i.e., a function $\zeta \in C_{0}^{\infty}(\mathbb{G})$ which is radially decreasing and is supported in $\{x \in \mathbb{G}:|x| \leq 1\}$ such that $\int \zeta d x=1$. Also, for $n \geq 1$ we denote by $\zeta_{n}$ the function defined by $\zeta_{n}(x)=n^{M} \zeta\left(\delta_{n}(x)\right)$, where $\delta_{n}$ is a dilation on $\mathbb{G}$ as defined in Section 2. The following theorem gives an existence result and global pointwise estimates for a quasilinear equation with measure data.

In what follows, for a finite measure $\mu$ defined in a bounded domain $\Omega \subset \mathbb{G}$, we will extend it by zero to $\mathbb{G} \backslash \Omega$ and explicitly define the convolution $\zeta_{n} * \mu$ by

$$
\zeta_{n} * \mu(x)=\int_{\mathbb{G}} \zeta_{n}\left(y^{-1} x\right) d \mu(y)=\int_{\Omega} \zeta_{n}\left(y^{-1} x\right) d \mu(y)
$$

for any $x \in \mathbb{G}$. It is then easy to see that $\zeta_{n} * \mu$ converges to $\mu$ weakly as measures in $\mathbb{G}$.

Theorem 4.1. Suppose that $\Omega$ is bounded and $\mu$ is a nonnegative finite measure on $\Omega$. Let $u_{n}$ be the unique solution in $S_{0}^{1, p}(\Omega)$ of

$$
-\Delta_{\mathbb{G}, p} u_{n}=\zeta_{n} * \mu \quad \text { in } \quad \Omega .
$$

Then there is a subsequence $\left\{u_{n_{k}}\right\}$ of $\left\{u_{n}\right\}$ and a p-superharmonic function $u$ on $\Omega$ such that

$$
u=\lim _{k \rightarrow \infty} u_{n_{k}} \quad \text { a.e. }
$$

Moreover, $u$ solves the equation

$$
\left\{\begin{array}{rll}
-\Delta_{\mathbb{G}, p} u & =\mu & \text { in } \Omega \\
u & =0 & \text { on } \partial \Omega
\end{array}\right.
$$

in the sense of Definition 1.1 .

Proof. Let $\mu_{n}=\zeta_{n} * \mu$, and let $v_{n}$ be the unique solution in $S_{0}^{1, p}(B)$ of

$$
-\Delta_{\mathbb{G}, p} v_{n}=\mu_{n} \quad \text { in } B,
$$

where $B=B(a, 2 R)$ with $R=\operatorname{diam}(\Omega)$ and $a \in \Omega$ so that $\Omega \subset B$. We now extend $u_{n}$ by zero outside $\Omega$ and set $\varphi=\min \left\{v_{n}-u_{n}, 0\right\}$. Since $\varphi \in S_{0}^{1, p}(\Omega) \cap S_{0}^{1, p}(B)$ we can use it as a test function in (4.1) and (4.3) to obtain

$$
\int_{B}\left|X v_{n}\right|^{p-2} X v_{n} \cdot X \varphi d x-\int_{\Omega}\left|X u_{n}\right|^{p-2} X u_{n} \cdot X \varphi d x=0
$$

or

$$
\int_{B \cap\left\{v_{n}<u_{n}\right\}}\left|X v_{n}\right|^{p-2} X v_{n} \cdot X \varphi d x-\int_{B \cap\left\{v_{n}<u_{n}\right\}}\left|X u_{n}\right|^{p-2} X u_{n} \cdot X \varphi d x=0 .
$$

This gives

$$
\int_{B \cap\left\{v_{n}<u_{n}\right\}}\left(\left|X v_{n}\right|^{p-2} X v_{n}-\left|X u_{n}\right|^{p-2} X u_{n}\right) \cdot X\left(v_{n}-u_{n}\right) d x=0 .
$$

Thus $\varphi=0$ a.e., or equivalently we have

$$
u_{n} \leq v_{n} \quad \text { a.e. }
$$


Since $\mu_{n}(\Omega) \leq \mu(\Omega)$, for each $k>0$, we have the estimate

$$
\begin{aligned}
\int_{\Omega}\left|X\left(\min \left\{u_{n}, k\right\}\right)\right|^{p} & =\int_{\Omega}\left|X u_{n}\right|^{p-2} X u_{n} \cdot X\left(\min \left\{u_{n}, k\right\}\right) \\
& =\int_{\Omega} \min \left\{u_{n}, k\right\} d \mu_{n} \leq k \mu_{n}(\Omega) \\
& \leq k \mu(\Omega) .
\end{aligned}
$$

Consequently, by Sobolev's embedding theorem we obtain

$$
\left\|\min \left\{u_{n}, k\right\}\right\|_{L^{\frac{M p}{M-p}}(\Omega)} \leq C(k \mu(\Omega))^{\frac{1}{p}} .
$$

Hence

$$
\begin{aligned}
\left|\left\{u_{n}>k\right\}\right| & \leq\left(\frac{1}{k}\left\|\min \left\{u_{n}, k\right\}\right\|_{L^{\frac{M p}{M-p}}(\Omega)}\right)^{\frac{M p}{M-p}} \\
& \leq C \mu(\Omega)^{\frac{M}{M-p}} k^{\frac{M(1-p)}{M-p}} .
\end{aligned}
$$

This gives

$$
\left\|u_{n}\right\|_{L^{p-1}(\Omega)} \leq|\Omega|^{\frac{p}{M(p-1)}}\left\|u_{n}\right\|_{L^{\frac{M(p-1)}{M-p}}, \infty} \leq C|\Omega|^{\frac{p}{M(p-1)}} \mu(\Omega)^{\frac{1}{p-1}} .
$$

Now, arguing as in KM1] we can find subsequences $\left\{u_{n_{k}}\right\},\left\{v_{n_{k}}\right\}$ and $p$-superharmonic functions $u, v$ on $\Omega$ such that $u_{n_{k}} \rightarrow u, v_{n_{k}} \rightarrow v$ a.e. Hence from (4.5) and Theorem 3.3 we see that $u$ is a distributional solution of (4.2). Similarly, $v$ also solves (4.2) in the distributional sense with $B$ in place of $\Omega$, and (4.5), (4.6) hold for $v_{n}$ with $B$ in place of $\Omega$ as well. In particular, this implies

$$
\|v\|_{L^{p-1}(B)} \leq C R^{\frac{p}{(p-1)}} \mu(\Omega)^{\frac{1}{p-1}} .
$$

Thus in view of (4.4) and Theorem 3.4 we get

$$
\begin{aligned}
u(x) & \leq v(x) \leq C \mathbf{W}_{1, p}^{\frac{2}{3} d(x)} \mu(x)+C \inf _{B_{\frac{1}{3} d(x)}(x)} v \\
& \leq C \mathbf{W}_{1, p}^{2 R} \mu(x)+C d(x)^{\frac{-M}{p-1}}\|v\|_{L^{p-1}(B)} \\
& \leq C \mathbf{W}_{1, p}^{2 R} \mu(x)+C R^{\frac{-M}{p-1}}\|v\|_{L^{p-1}(B)},
\end{aligned}
$$

where $x \in \Omega$ and $d(x)=\operatorname{dist}(x, \partial B)$. Note that we have used the fact that $d(x) \geq R$ in the last inequality. Finally, from this and (4.7) we obtain the pointwise estimate

$$
u(x) \leq C \mathbf{W}_{1, p}^{2 \operatorname{diam}(\Omega)}(x)
$$

for all $x \in \Omega$. Thus $u$ solves (4.2) in the potential theoretic sense and the proof is complete.

Corollary 4.2. For any nonnegative finite measure $\mu$ on $\Omega$, there exists a potential theoretic solution to equation (1.7).

We now construct a solution to a nonlinear equation with a power source term under a certain iterated Wolff's potential condition. This condition turns out to be sharp, as we will see later.

Theorem 4.3. Let $\omega$ be a nonnegative finite measure on $\Omega$. Let $p>1$ and $q>p-1$. Suppose that $R=\operatorname{diam}(\Omega)$, and

$$
\mathbf{W}_{1, p}^{2 R}\left(\mathbf{W}_{1, p}^{2 R} \omega\right)^{q} \leq C \mathbf{W}_{1, p}^{2 R} \omega \text { a.e. },
$$


where

$$
C \leq\left(\frac{q-p+1}{q A \max \left\{1,2^{p^{\prime}-2}\right\}}\right)^{q\left(p^{\prime}-1\right)}\left(\frac{p-1}{q-p+1}\right),
$$

and $A$ is the constant in Definition 1.1 . Then there is a solution $u \in L^{q}(\Omega)$ to the equation

$$
\left\{\begin{aligned}
-\Delta_{\mathbb{G}, p} u & =u^{q}+\omega \text { in } \Omega \\
u & =0 \text { on } \partial \Omega .
\end{aligned}\right.
$$

Moreover, for every $x$ in $\Omega$,

$$
u(x) \leq \kappa \mathbf{W}_{1, p}^{2 R} \omega(x),
$$

where the constant $\kappa$ depends only on $p, q, M$.

Proof. Let $u_{n}^{(1)}$ solve the equation

$$
\left\{\begin{aligned}
-\Delta_{\mathbb{G}, p} u_{n}^{(1)} & =\zeta_{n} * \omega \quad \text { in } \Omega \\
u_{n}^{(1)} & =0 \text { on } \partial \Omega .
\end{aligned}\right.
$$

By Theorem 4.1, there exists a function $u^{(1)}$ that satisfies

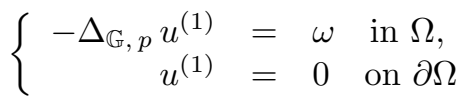

in the sense of Definition 1.1, and for a subsequence of $\left\{u_{n}^{(1)}\right\}$, still denoted by $\left\{u_{n}^{(1)}\right\}$, we have

$$
u^{(1)}=\lim _{n \rightarrow \infty} u_{n}^{(1)} \quad \text { a.e. }
$$

Similarly, let $u_{n}^{(2)}$ be a solution to the equation

$$
\left\{\begin{aligned}
-\Delta_{\mathbb{G}, p} u_{n}^{(2)} & =\zeta_{n} *\left[u^{(1)}\right]^{q}+\zeta_{n} * \omega & \text { in } \Omega, \\
u_{n}^{(2)} & = & 0 \text { on } \partial \Omega .
\end{aligned}\right.
$$

Then by Theorem 4.1, there exists a function $u^{(2)}$ that satisfies

$$
\left\{\begin{aligned}
-\Delta_{\mathbb{G}, p} u^{(2)} & =\left[u^{(1)}\right]^{q}+\omega \quad \text { in } \Omega, \\
u^{(2)} & =0 \quad \text { on } \partial \Omega
\end{aligned}\right.
$$

in the sense of Definition 1.1, and for a subsequence of $\left\{u_{n}^{(2)}\right\}$, still denoted by $\left\{u_{n}^{(2)}\right\}$, we have

$$
u^{(2)}=\lim _{n \rightarrow \infty} u_{n}^{(2)} \text { a.e. }
$$

As $u_{n}^{(1)} \leq u_{n}^{(2)}$ we see from (4.12) and (4.13) that $u^{(1)} \leq u^{(2)}$ a.e. and hence everywhere since they are $p$-superharmonic. Thus by induction we can find an increasing sequence $\left\{u^{(k)}\right\}$ such that $u^{(1)}$ satisfies (4.11) and for $k \geq 2$,

$$
\left\{\begin{aligned}
-\Delta_{\mathbb{G}, p} u^{(k)} & =\left[u^{(k-1)}\right]^{q}+\omega \text { in } \Omega, \\
u^{(k)} & =0 \text { on } \partial \Omega
\end{aligned}\right.
$$

in the sense of Definition 1.1. Note that we have

$$
u^{(1)} \leq A \mathrm{~W}_{1, p}^{2 R} \omega, \quad u^{(k)} \leq A \mathrm{~W}_{1, p}^{2 R}\left(\left[u^{(k-1)}\right]^{q}+\omega\right)
$$


for all $k \geq 2$. In view of these estimates and the condition (4.8) we get

$$
\begin{aligned}
u^{(2)} & \leq A \max \left\{1,2^{p^{\prime}-2}\right\}\left[\mathbf{W}_{1, p}^{2 R}\left[u^{(1)}\right]^{q}+\mathbf{W}_{1, p}^{2 R} \omega\right] \\
& \leq A \max \left\{1,2^{p^{\prime}-2}\right\}\left(c_{1}^{q\left(p^{\prime}-1\right)} C+1\right) \mathbf{W}_{1, p}^{2 R} \omega \\
& =c_{2} \mathbf{W}_{1, p}^{2 R} \omega,
\end{aligned}
$$

where $c_{1}=A$ and $c_{2}=A \max \left\{1,2^{p^{\prime}-2}\right\}\left(c_{1}^{q\left(p^{\prime}-1\right)} C+1\right)$. By induction we can find a sequence $\left\{c_{k}\right\}_{k \geq 1}$ of positive numbers such that

$$
u^{(k)} \leq c_{k} \mathbf{W}_{1, p}^{2 R} \omega
$$

with

$$
c_{k}=A \max \left\{1,2^{p^{\prime}-2}\right\}\left(c_{k-1}^{q\left(p^{\prime}-1\right)} C+1\right)
$$

for all $k \geq 2$. It is then easy to see that $c_{k} \leq \frac{A \max \left\{1,2^{p^{\prime}-2}\right\} q}{q-p+1}$ for all $k \geq 1$ as long as $C$ satisfies (4.9). Thus

$$
u^{(k)} \leq \frac{A \max \left\{1,2^{p^{\prime}-2}\right\} q}{q-p+1} \mathbf{W}_{1, p}^{2 R} \omega .
$$

Therefore, $\left\{u^{(k)}\right\}$ converges pointwise increasingly to a nonnegative function $u$ for which

$$
u \leq \kappa \mathrm{W}_{1, p}^{2 R} \omega
$$

Finally, in view of (4.14) and Theorem [3.3, we see that $u$ solves (4.10) in the sense of Definition 1.1. This completes the proof of the theorem.

In the general context of homogeneous spaces, it was proved in $[\mathrm{SW}$ and $\mathrm{Chr}$. that for $\lambda=8$, and for any (large negative) integer $m$, there are points $\left\{x_{j}^{k}\right\} \subset \mathbb{G}$ and a family of sets $\mathcal{D}_{m}=\left\{E_{j}^{k}\right\}, k=m, m+1, \ldots$ and $j=1,2, \ldots$ such that

(i) $B_{\lambda^{k}}\left(x_{j}^{k}\right) \subset E_{j}^{k} \subset B_{\lambda^{k+1}}\left(x_{j}^{k}\right)$;

(ii) for each fixed $k=m, m+1, \ldots$, the sets $E_{j}^{k}$ are pairwise disjoint in $j$, and

$$
\mathbb{G}=\bigcup_{j \geq 1} E_{j}^{k}
$$

(iii) if $k<l$, then either $E_{j}^{k} \cap E_{i}^{l}=\emptyset$ or $E_{j}^{k} \subset E_{i}^{l}$.

We shall say that the family $\mathcal{D}=\bigcup_{m=-\infty}^{\infty} \mathcal{D}_{m}$ is a dyadic cube decomposition of $\mathbb{G}$, and call sets in $\mathcal{D}$ dyadic cubes and denote them by $Q$. Note that the cubes in $\mathcal{D}_{m_{1}}$ may have no relation to those in $\mathcal{D}_{m_{2}}$ if $m_{1}$ and $m_{2}$ are different. If $Q=E_{j}^{k} \in \mathcal{D}_{m}$ for some $m$, we say $Q$ is centered at $x_{j}^{k}$ and define the side length of $Q$ to be $\ell(Q)=\lambda^{k}$. We also denote by $Q^{*}$ the ball $B_{\lambda^{k+1}}\left(x_{j}^{k}\right)$ containing $Q$ and by $Q^{* *}$ the ball $B_{2 \lambda^{k+2}}\left(x_{j}^{k}\right)$.

Remark 4.4. Note that if $Q^{* *}=B_{2 \lambda^{k+2}}\left(x_{j_{1}}^{k}\right)$ and if $\left\{E_{j_{i}}^{k}\right\}, i=1, \ldots, d$, are the dyadic cubes that intersect $Q^{* *}$, then obviously $Q^{* *} \subset \bigcup_{i=1}^{m} E_{j_{i}}^{k}$. Moreover, since each $E_{j_{i}}^{k} \subset B_{4 \lambda^{k+2}}\left(x_{j_{1}}^{k}\right)$, we obtain

$$
c d \lambda^{k M} \leq\left|\bigcup_{i=1}^{d} E_{j_{i}}^{k}\right| \leq\left|B_{4 \lambda^{k+2}}\left(x_{j_{1}}^{k}\right)\right| \leq C \lambda^{k M},
$$

which gives

$$
d \leq C=C(\mathbb{G})
$$


This implies that the ball $Q^{* *}=B_{2 \lambda^{k+2}}\left(x_{j_{1}}^{k}\right)$ is contained in the union of at most $d$ dyadic cubes of side length $\lambda^{k}$ for some constant $d=d(\mathbb{G})$.

For an integer $m$, let $\Lambda=\left\{\lambda_{Q}\right\}_{Q \in \mathcal{D}_{m}}, \lambda_{Q} \geq 0$, and let $\sigma$ be a positive locally finite Borel measure on $\mathbb{G}$ such that $\lambda_{Q}=0$ whenever $\sigma(Q)=0$. We will follow the convention that $0 \cdot \infty=0$. For $1<s<+\infty$, we define

$$
\begin{aligned}
& A_{1}^{m}(\Lambda)=\int_{\mathbb{G}}\left[\sum_{Q \in \mathcal{D}_{m}} \frac{\lambda_{Q}}{\sigma(Q)} \chi_{Q}(x)\right]^{s} d \sigma(x), \\
& A_{2}^{m}(\Lambda)=\sum_{Q \in \mathcal{D}_{m}} \lambda_{Q}\left[\frac{1}{\sigma(Q)} \sum_{Q^{\prime} \in \mathcal{D}_{m}, Q^{\prime} \subset Q} \lambda_{Q^{\prime}}\right]^{s-1}, \\
& A_{3}^{m}(\Lambda)=\int_{\mathbb{G} x \in Q \subset \mathcal{D}_{m}} \sup _{\sigma(Q)}\left[\frac{1}{\sum_{Q^{\prime} \in \mathcal{D}_{m}, Q^{\prime} \subset Q}} \lambda_{Q^{\prime}}\right]^{s} d \sigma(x) .
\end{aligned}
$$

The proof of the following proposition will be omitted as it is similar to the one given in $[\mathrm{COV}]$ in the case $\mathbb{G}=\mathbb{R}^{N}$ and $\mathcal{D}_{m}$ is the set of all standard dyadic cubes in $\mathbb{R}^{N}$.

Proposition 4.5. Let $\sigma$ be a positive locally finite Borel measure on $\mathbb{G}$. Let $1<$ $s<+\infty$. Then there exist constants $C_{i}>0, i=1,2,3$, which depend only on $s$, such that

$$
A_{1}^{m}(\Lambda) \leq C_{1} A_{2}^{m}(\Lambda) \leq C_{2} A_{3}^{m}(\Lambda) \leq C_{3} A_{1}^{m}(\Lambda)
$$

for all $\Lambda=\left\{\lambda_{Q}\right\}_{Q \in \mathcal{D}_{m}}$ with $\lambda_{Q} \geq 0$, and $m \in \mathbb{Z}$.

We next consider the following quantities. For each integer $m$, a dyadic cube $P \in \mathcal{D}_{m}$, and a nonnegative Borel measure $\mu$ on $\mathbb{G}$, we define

$$
\begin{aligned}
& \text { (a) } B_{1}^{m}(P, \mu)=\sum_{Q \subset P}\left[\frac{\mu(Q)}{|Q|^{1-\frac{\alpha p}{Q}}}\right]^{\frac{q}{p-1}}|Q| \\
& \text { (b) } B_{2}^{m}(P, \mu)=\int_{P}\left[\sum_{Q \subset P} \frac{\mu(Q)^{\frac{1}{p-1}}}{|Q|^{\left(1-\frac{\alpha p}{Q}\right) \frac{1}{p-1}}} \chi_{Q}(x)\right]^{q} d x \\
& \text { (c) } B_{3}^{m}(P, \mu)=\int_{P}\left[\sum_{Q \subset P} \frac{\mu(Q)}{|Q|^{1-\frac{\alpha p}{Q}}} \chi_{Q}(x)\right]^{\frac{q}{p-1}} d x .
\end{aligned}
$$

Here $\alpha>0, p>1, q>p-1$, and the sum is taken over all dyadic cubes $Q \in \mathcal{D}_{m}$ such that $Q \subset P$.

Proposition 4.6. There exist constants $C_{i}>0, i=1,2,3$, independent of $m, P$, and $\mu$ such that

$$
B_{1}^{m}(P, \mu) \leq C_{1} B_{2}^{m}(P, \mu) \leq C_{2} B_{3}^{m}(P, \mu) \leq C_{3} B_{1}^{m}(P, \mu)
$$


Proof. Let $\Lambda=\left\{\lambda_{Q}\right\}_{Q \in \mathcal{D}_{m}}$ where $\lambda_{Q}=\mu(Q)|Q|^{\frac{\alpha p}{M}}$ if $Q \subset P$ and $\lambda_{Q}=0$ otherwise. Applying Proposition 4.5 with $d \sigma=\chi_{P} d x$ and $s=\frac{q}{p-1}>1$, we obtain

$$
\begin{aligned}
B_{3}^{m}(P, \mu) & \leq C \sum_{Q \subset P} \lambda_{Q}\left[\frac{1}{|Q|} \sum_{Q^{\prime} \subset Q} \lambda_{Q^{\prime}}\right]^{\frac{q}{p-1}-1} \\
& =C \sum_{Q \subset P} \mu(Q)|Q|^{\frac{\alpha p}{M}}\left[\frac{1}{|Q|} \sum_{Q^{\prime} \subset Q} \mu\left(Q^{\prime}\right)\left|Q^{\prime}\right|^{\frac{\alpha p}{M}}\right]^{\frac{q}{p-1}-1} \\
& \leq C \sum_{Q \subset P}\left[\frac{\mu(Q)}{|Q|^{1-\frac{\alpha p}{M}}}\right]^{\frac{q}{p-1}}|Q|=C B_{1}^{m}(P, \mu) .
\end{aligned}
$$

Furthermore, since $\frac{q}{p-1}>1$,

$$
\begin{aligned}
B_{1}^{m}(P, \mu) & =\int_{P} \sum_{Q \subset P}\left[\frac{\mu(Q)}{|Q|^{1-\frac{\alpha p}{M}}}\right]^{\frac{q}{p-1}} \chi_{Q}(x) d x \leq B_{3}^{m}(P, \mu) \\
& \leq C \int_{P} \sup _{x \in Q \subset P}\left[\frac{1}{|Q|} \sum_{Q^{\prime} \subset Q} \lambda_{Q^{\prime}}\right]^{\frac{q}{p-1}} d x \\
& \leq C \int_{P} \sup _{x \in Q \subset P}\left[\frac{\mu(Q)}{|Q|^{1-\frac{\alpha p}{M}}}\right]^{\frac{q}{p-1}} d x \leq C B_{2}^{m}(P, \mu),
\end{aligned}
$$

where we have used Proposition 4.5 in the second inequality. We next observe that for $p \leq 2, B_{2}^{m}(P, \mu) \leq B_{3}^{m}(P, \mu)$. Thus it remains to show that for $p>2$, $B_{2}^{m}(P, \mu) \leq C B_{3}^{m}(P, \mu)$. Since $q>p-1>1$, by Proposition 4.5 we have

$$
\begin{aligned}
B_{2}^{m}(P, \mu) & =\int_{P}\left[\sum_{Q \subset P} \frac{\mu(Q)^{\frac{1}{p-1}}}{|Q|^{\left(1-\frac{\alpha p}{M}\right) \frac{1}{p-1}}} \chi_{Q}(x)\right]^{q} d x \\
& \leq C \sum_{Q \subset P} \frac{\mu(Q)^{\frac{1}{p-1}}}{|Q|^{\left(1-\frac{\alpha p}{M}\right) \frac{1}{p-1}+q-2}}\left[\sum_{Q^{\prime} \subset Q} \frac{\mu\left(Q^{\prime}\right)^{\frac{1}{p-1}}}{\left|Q^{\prime}\right|^{\left(1-\frac{\alpha p}{M}\right) \frac{1}{p-1}-1}}\right]^{q-1} .
\end{aligned}
$$

On the other hand, by Hölder's inequality the sum in the above square brackets can be estimated by

$$
\begin{aligned}
& \sum_{Q^{\prime} \subset Q}\left(\mu\left(Q^{\prime}\right)^{\frac{1}{p-1}}\left|Q^{\prime}\right|^{\epsilon}\right)\left|Q^{\prime}\right|^{-\left(1-\frac{\alpha p}{M}\right) \frac{1}{p-1}+1-\epsilon} \\
& \leq\left(\sum_{Q^{\prime} \subset Q} \mu\left(Q^{\prime}\right)^{\frac{r^{\prime}}{p-1}}\left|Q^{\prime}\right|^{\epsilon r^{\prime}}\right)^{\frac{1}{r^{\prime}}}\left(\sum_{Q^{\prime} \subset Q}\left|Q^{\prime}\right|^{-r\left(1-\frac{\alpha p}{M}\right) \frac{1}{p-1}+r-r \epsilon}\right)^{\frac{1}{r}},
\end{aligned}
$$

where $r^{\prime}=p-1>1, r=\frac{p-1}{p-2}$, and $\epsilon>0$ is chosen so that $-r\left(1-\frac{\alpha p}{M}\right) \frac{1}{p-1}+r-r \epsilon>1$, i.e., $0<\epsilon<\frac{\alpha p}{(p-1) M}$. Therefore,

$$
\begin{aligned}
\sum_{Q^{\prime} \subset Q} \frac{\mu\left(Q^{\prime}\right)^{\frac{1}{p-1}}}{\left|Q^{\prime}\right|^{\left(1-\frac{\alpha p}{M}\right) \frac{1}{p-1}-1}} & \leq C \mu(Q)^{\frac{1}{p-1}}|Q|^{\epsilon}|Q|^{-\left(1-\frac{\alpha p}{M}\right) \frac{1}{p-1}+1-\epsilon} \\
& =C \frac{\mu(Q)^{\frac{1}{p-1}}}{|Q|^{\left(1-\frac{\alpha p}{M}\right) \frac{1}{p-1}-1}} .
\end{aligned}
$$


Hence, combining the preceding inequalities, we obtain

$$
\begin{aligned}
B_{2}^{m}(P, \mu) & \leq C \sum_{Q \subset P} \frac{\mu(Q)^{\frac{1}{p-1}}}{|Q|^{\left(1-\frac{\alpha p}{M}\right) \frac{1}{p-1}+q-2}}\left[\frac{\mu(Q)^{\frac{1}{p-1}}}{|Q|^{\left(1-\frac{\alpha p}{M}\right) \frac{1}{p-1}-1}}\right]^{q-1} \\
& =C \sum_{Q \subset P} \frac{\mu(Q)^{\frac{q}{p-1}}}{|Q|^{\left(1-\frac{\alpha p}{M}\right) \frac{q}{p-1}-1}}=C B_{1}^{m}(P, \mu) \leq C B_{3}^{m}(P, \mu) .
\end{aligned}
$$

This completes the proof of the proposition.

Remark 4.7. From Remark 4.4 we see that

$$
\begin{aligned}
\sum_{Q^{\prime} \subset Q} \mu\left(Q^{\prime * *}\right)\left|Q^{\prime}\right|^{\beta} & =\sum_{k=0}^{\infty} \sum_{\substack{\left.\ell Q^{\prime}\right)=\ell(Q) / \lambda^{k}, Q^{\prime} \subset Q}} \mu\left(Q^{\prime * *}\right)\left|Q^{\prime}\right|^{\beta} \\
& \leq C \sum_{k=0}^{\infty} \lambda^{-k \beta M} \ell(Q)^{\beta M} \sum_{\substack{\ell\left(Q^{\prime}\right)=\ell(Q) / \lambda^{k}, Q^{\prime} \subset Q}} \mu\left(Q^{\prime * *}\right) \\
& \leq C \mu\left(Q^{* *}\right)|Q|^{\beta}
\end{aligned}
$$

for any $\beta>0$. Thus the chain of inequalities in (4.15) still holds if $\mu(Q)$ is replaced by $\mu\left(Q^{* *}\right)$ in the definition of $B_{i}^{m}(P, \mu), i=1,2,3$.

Lemma 4.8. Let $\alpha>0$ and $p>1$. Then for any integer $m$,

$$
\mathbf{W}_{\alpha, p}^{r} \mu(x) \geq c \sum_{\substack{Q \in \mathcal{D}_{m}, \ell(Q) \leq \lambda^{-3} r}}\left[\frac{\mu(Q)}{|Q|^{1-\frac{\alpha p}{M}}}\right]^{\frac{1}{p-1}} \chi_{Q}(x),
$$

and

$$
\int_{\lambda^{m} r}^{r}\left[\frac{\mu\left(B_{t}(x)\right)}{t^{M-\alpha p}}\right]^{\frac{1}{p-1}} \frac{d t}{t} \leq C \sum_{\substack{Q \in \mathcal{D}_{m+\left[\log _{\lambda} r\right]}, \ell(Q) \leq r}}\left[\frac{\mu\left(Q^{* *}\right)}{|Q|^{1-\frac{\alpha p}{M}}}\right]^{\frac{1}{p-1}} \chi_{Q}(x) .
$$

In (4.17) $\left[\log _{\lambda} r\right]$ stands for the integral part of the real number $\log _{\lambda} r$.

Proof. To prove (4.16) we may assume that $\lambda^{m} \leq \lambda^{-3} r$ since dyadic cubes in $\mathcal{D}_{m}$ have side length not smaller than $\lambda^{m}$. Then $\left[\log _{\lambda} r\right]-3 \geq m$. Observe that

$$
\begin{aligned}
& \mathbf{W}_{\alpha, p}^{r} \mu(x)=\sum_{k=0}^{\infty} \int_{\lambda^{-k-1} r}^{\lambda^{-k} r}\left[\frac{\mu\left(B_{t}(x)\right)}{t^{M-\alpha p}}\right]^{\frac{1}{p-1}} \frac{d t}{t} \\
& \geq c \sum_{k=0}^{\infty}\left[\frac{\mu\left(B_{\lambda^{-k-1} r}(x)\right)}{\left(\lambda^{-k-1} r\right)^{M-\alpha p}}\right]^{\frac{1}{p-1}} \\
& \geq c \sum_{k=0}^{\infty} \sum_{E_{j}^{-k-3+\left[\log _{\lambda} r\right]} \in \mathcal{D}_{m}}\left[\frac{\mu\left(E_{j}^{-k-3+\left[\log _{\lambda} r\right]}\right)}{\ell\left(E_{j}^{-k-3+\left[\log _{\lambda} r\right]}\right)^{M-\alpha p}}\right] \chi_{E_{j}^{-k-3+\left[\log _{\lambda} r\right]}(x),}
\end{aligned}
$$

where the last inequality follows from the fact that for $k \geq 0$ and $x \in E_{j}^{-k-3+\left[\log _{\lambda} r\right]} \in$ $\mathcal{D}_{m}$,

$$
E_{j}^{-k-3+\left[\log _{\lambda} r\right]} \subset B_{\lambda^{-k-2+\left[\log _{\lambda} r\right]}}\left(x_{j}^{-k-3+\left[\log _{\lambda} r\right]}\right) \subset B_{\lambda^{-k-1} r}(x) .
$$


Thus we obtain (4.16). Similarly, to prove (4.17) we may assume that $m<0$, and we have

$$
\begin{aligned}
& \int_{\lambda^{m} r}^{r}\left[\frac{\mu\left(B_{t}(x)\right)}{t^{M-\alpha p}}\right]^{\frac{1}{p-1}} \frac{d t}{t}=\sum_{k=0}^{|m|-1} \int_{\lambda^{-k-1} r}^{\lambda^{-k} r}\left[\frac{\mu\left(B_{t}(x)\right)}{t^{M-\alpha p}}\right]^{\frac{1}{p-1}} \frac{d t}{t} \\
& \leq C \sum_{k=0}^{|m|-1}\left[\frac{\mu\left(B_{\lambda^{-k} r}(x)\right)}{\left(\lambda^{-k} r\right)^{M-\alpha p}}\right]^{\frac{1}{p-1}} \\
& \leq C \sum_{k=0}^{|m|-1} \sum_{j}\left[\frac{\mu\left(B_{2 \lambda^{-k} r}\left(x_{j}^{-k-1+\left[\log _{\lambda} r\right]}\right)\right)}{\left(\lambda^{-k} r\right)^{M-\alpha p}}\right]^{\frac{1}{p-1}} \chi_{E_{j}^{-k-1+\left[\log _{\lambda} r\right]}(x) .}
\end{aligned}
$$

Here $E_{j}^{-k-1+\left[\log _{\lambda} r\right]} \in D_{m+\left[\log _{\lambda} r\right]}$, and the last inequality follows since for $x \in$ $E_{j}^{-k-1+\left[\log _{\lambda} r\right]}$, we have $x \in B_{\lambda^{-k+\left[\log _{\lambda} r\right]}}\left(x_{j}^{-k-1+\left[\log _{\lambda} r\right]}\right)$ and hence

$$
B_{\lambda^{-k} r}(x) \subset B_{2 \lambda^{-k} r}\left(x_{j}^{-k-1+\left[\log _{\lambda} r\right]}\right) .
$$

This gives (4.17) and completes the proof of the lemma.

The result obtained in the following theorem may be considered as an analogue of Wolff's inequality (see [HW], PV1]) which is crucial in our approach to quasilinear Lane-Emden type equations later on.

Theorem 4.9. Let $\alpha>0, p>1$, and $q>p-1$. Then for any $0<r<\infty$ and any nonnegative Borel measure $\mu$ on $\mathbb{G}$,

$$
\int_{\mathbb{G}}\left[\mathbf{W}_{\alpha, p}^{r} \mu(x)\right]^{q} d x \cong \sup _{m \in \mathbb{Z}} \sum_{Q \in \mathcal{D}_{m}, \ell(Q) \leq r}\left[\frac{\mu(Q)}{|Q|^{1-\frac{\alpha p}{M}}}\right]^{\frac{q}{p-1}}|Q|,
$$

where the constants of equivalence are independent of $r$ and $\mu$.

Proof. Let $k \in \mathbb{Z}$ be such that $\frac{r}{\lambda}<\lambda^{k} \leq r$. For any interger $m \leq 0$, by Lemma 4.8 we have

$$
\begin{aligned}
& \int_{\mathbb{G}}\left\{\int_{\lambda^{m} r}^{r}\left[\frac{\mu\left(B_{t}(x)\right)}{t^{M-\alpha p}}\right]^{\frac{1}{p-1}} \frac{d t}{t}\right\}^{q} d x \\
& =\sum_{j: E_{j}^{k} \in \mathcal{D}_{m+\left[\log _{\lambda} r\right]}} \int_{E_{j}^{k}}\left\{\int_{\lambda^{m} r}^{r}\left[\frac{\mu\left(B_{t}(x)\right)}{t^{M-\alpha p}}\right]^{\frac{1}{p-1}} \frac{d t}{t}\right\}^{q} d x \\
& \leq C \sum_{j: E_{j}^{k} \in \mathcal{D}_{m+\left[\log _{\lambda} r\right]}} \int_{E_{j}^{k}}\left\{\sum_{\substack{Q \in \mathcal{D}_{m+\left[\log _{\lambda} r\right]}, \ell(Q) \leq r}}\left[\frac{\mu\left(Q^{* *}\right)}{|Q|^{1-\frac{\alpha p}{M}}}\right]^{\frac{1}{p-1}} \chi_{Q}(x)\right\}^{q} d x \\
& =C \sum_{j: E_{j}^{k} \in \mathcal{D}_{m+\left[\log _{\lambda} r\right]}} \int_{E_{j}^{k}}\left\{\sum_{\substack{Q \in \mathcal{D}_{m+\left[\log _{\lambda} r\right]}, Q \subset E_{j}^{k}}}\left[\frac{\mu\left(Q^{* *}\right)}{|Q|^{1-\frac{\alpha p}{M}}}\right]^{\frac{1}{p-1}} \chi_{Q}(x)\right\}^{q} d x .
\end{aligned}
$$


Thus by Proposition 4.6 and Remark 4.7 we obtain

$$
\begin{aligned}
& \int_{\mathbb{G}}\left\{\int_{\lambda^{m} r}^{r}\left[\frac{\mu\left(B_{t}(x)\right)}{t^{M-\alpha p}}\right]^{\frac{1}{p-1}} \frac{d t}{t}\right\}^{q} d x \\
& \leq \quad C \sum_{j: E_{j}^{k} \in \mathcal{D}_{m+\left[\log _{\lambda} r\right]}} \sum_{\substack{Q \in \mathcal{D}_{m+\left[\log _{\lambda} r\right]}, Q \subset E_{j}^{k}}}\left[\frac{\mu\left(Q^{* *}\right)}{|Q|^{1-\frac{\alpha p}{M}}}\right]^{\frac{q}{p-1}}|Q| \\
& =C \sum_{\substack{Q \in \mathcal{D}_{m+\left[\log _{\lambda} r\right]}, \ell(Q) \leq r}}\left[\frac{\mu\left(Q^{* *}\right)}{|Q|^{1-\frac{\alpha p}{M}}}\right]^{\frac{q}{p-1}}|Q| \\
& \leq C \sum_{\substack{Q \in \mathcal{D}_{m+\left[\log _{\lambda} r\right]}, \ell(Q) \leq r}}\left[\frac{\mu(Q)}{|Q|^{1-\frac{\alpha p}{M}}}\right]^{\frac{q}{p-1}}|Q|,
\end{aligned}
$$

where the last inequality follows from Remark 4.4. This gives

$$
\begin{aligned}
\int_{\mathbb{G}}\left[\mathbf{W}_{\alpha, p}^{r} \mu(x)\right]^{q} d x & \leq C \sup _{m \in \mathbb{Z}} \sum_{\substack{Q \in \mathcal{D}_{m+\left[l \log _{\lambda} r\right]}, \ell(Q) \leq r}}\left[\frac{\mu(Q)}{|Q|^{1-\frac{\alpha p}{M}}}\right]^{\frac{q}{p-1}}|Q| \\
& \leq C \sup _{m \in \mathbb{Z}} \sum_{Q \in \mathcal{D}_{m}, \ell(Q) \leq r}\left[\frac{\mu(Q)}{|Q|^{1-\frac{\alpha p}{M}}}\right]^{\frac{q}{p-1}}|Q| .
\end{aligned}
$$

Analogously, from (4.16) in Lemma 4.8 we obtain

$$
\int_{\mathbb{G}}\left[\mathbf{W}_{\alpha, p}^{\lambda^{3} r} \mu(x)\right]^{q} d x \geq C \sup _{m \in \mathbb{Z}} \sum_{Q \in \mathcal{D}_{m}, \ell(Q) \leq r}\left[\frac{\mu(Q)}{|Q|^{1-\frac{\alpha p}{M}}}\right]^{\frac{q}{p-1}}|Q| .
$$

Note that

$$
\int_{\mathbb{G}}\left[\mathbf{W}_{\alpha, p}^{\lambda^{3} r} \mu(x)\right]^{q} d x \leq C \int_{\mathbb{G}}\left[\mathbf{W}_{\alpha, p}^{r} \mu(x)\right]^{q} d x
$$

if we can show that

$$
\int_{\mathbb{G}}\left[\frac{\mu\left(B_{\lambda^{3} r}(x)\right)}{r^{M-\alpha p}}\right]^{\frac{q}{p-1}} d x \leq C \int_{\mathbb{G}}\left[\mathbf{W}_{\alpha, p}^{r} \mu(x)\right]^{q} d x .
$$

To prove (4.18), we choose an interger $k$ so that $\lambda^{k+1}<\frac{r}{4} \leq \lambda^{k+2}$ and as in Remark 4.4, it can be seen that for $x \in E_{j_{1}}^{k} \subset \mathcal{D}_{k}$ for some $j_{1} \geq 1$ the ball $B_{\lambda^{3} r}(x)$ is contained in the union of at most $d$ cubes in $\left\{E_{j}^{k}\right\}_{j \geq 1} \subset \mathcal{D}_{k}$ for some constant $d=d(\mathbb{G})$. That is,

$$
B_{\lambda^{3} r}(x) \subset \bigcup_{i=1}^{d} E_{j_{i}}^{k}
$$


Thus we obtain

$$
\begin{aligned}
\int_{\mathbb{G}} \mu\left(B_{\lambda^{3} r}(x)\right)^{\frac{q}{p-1}} d x & =\sum_{j} \int_{E_{j}^{k}} \mu\left(B_{\lambda^{3} r}(x)\right)^{\frac{q}{p-1}} d x \\
& \leq C \sum_{j} \int_{E_{j}^{k}}\left[\mu\left(E_{j_{1}}^{k}\right)^{\frac{q}{p-1}}+\cdots+\mu\left(E_{j_{d}}^{k}\right)^{\frac{q}{p-1}}\right] d x \\
& \leq C \sum_{j}\left[\int_{E_{j_{1}}^{k}} \mu\left(E_{j_{1}}^{k}\right)^{\frac{q}{p-1}}+\cdots+\int_{E_{j_{d}}^{k}} \mu\left(E_{j_{d}}^{k}\right)^{\frac{q}{p-1}}\right] d x \\
& \leq C \sum_{j} \int_{E_{j}^{k}} \mu\left(E_{j}^{k}\right)^{\frac{q}{p-1}} d x .
\end{aligned}
$$

Therefore, we get

$$
\begin{aligned}
\int_{\mathbb{G}}\left[\frac{\mu\left(B_{\lambda^{3} r}(x)\right)}{r^{M-\alpha p}}\right]^{\frac{q}{p-1}} d x & \leq C \sum_{j} \int_{E_{j}^{k}}\left[\frac{\mu\left(B_{\frac{r}{2}}(x)\right)}{r^{M-\alpha p}}\right]^{\frac{q}{p-1}} d x \\
& \leq C \sum_{j} \int_{E_{j}^{k}}\left\{\int_{0}^{r}\left[\frac{\mu\left(B_{t}(x)\right)}{t^{M-\alpha p}}\right]^{\frac{1}{p-1}} \frac{d t}{t}\right\}^{q} d x
\end{aligned}
$$

which gives (4.18) and completes the proof of the theorem.

We also have a continuous version of Wolff's inequality which is known in the standard Euclidean setting [PV1].

Theorem 4.10. Let $\alpha>0, p>1$, and $q>p-1$. Then for any $0<r<\infty$ and any nonnegative Borel measure $\mu$ on $\mathbb{G}$,

$$
\left\|\mathbf{W}_{\alpha, p}^{r} \mu\right\|_{L^{q}(d x)}^{q} \cong\left\|\mathbf{W}_{\alpha p, \frac{q}{q-p+1}}^{r} \mu\right\|_{L^{1}(d \mu)} \cong\left\|\mathbf{G}_{\alpha p} \mu\right\|_{L^{\frac{q}{p-1}}(d x)}^{\frac{q}{p-1}},
$$

where the constants in these equivalences are independent $\mu$.

Proof. By arguing as in the proof of Theorem 4.9 we also find that

$$
\left\|\mathbf{W}_{\alpha p, \frac{q}{q-p+1}}^{r} \mu\right\|_{L^{1}(d \mu)} \cong \sup _{m \in \mathbb{Z}} \sum_{Q \in \mathcal{D}_{m}, \ell(Q) \leq r}\left[\frac{\mu(Q)}{|Q|^{1-\frac{\alpha p}{M}}}\right]^{\frac{q}{p-1}}|Q| .
$$

Thus by Theorem 4.9,

$$
\left\|\mathbf{W}_{\alpha, p}^{r} \mu\right\|_{L^{q}(d x)}^{q} \cong\left\|\mathbf{W}_{\alpha p, \frac{q}{q-p+1}}^{r} \mu\right\|_{L^{1}(d \mu)} .
$$

On the other hand, by Wolff's inequality (see [CLL], and [AH], Tu] in the Euclidean setting),

which gives the theorem.

$$
\left\|\mathbf{W}_{\alpha p, \frac{q}{q-p+1}}^{r} \mu\right\|_{L^{1}(d \mu)} \cong\left\|\mathbf{G}_{\alpha p} \mu\right\|_{L^{\frac{q}{p-1}}(d x)}^{\frac{q}{p-1}},
$$

Similarly, in the case $r=\infty$ we have the following Wolff type inequality.

Theorem 4.11. Let $\alpha>0,1<p<M / \alpha$ and $q>p-1$. Then for any nonnegative Borel measure $\mu$ on $\mathbb{G}$,

$$
\left\|\mathbf{W}_{\alpha, p}^{\infty} \mu\right\|_{L^{q}(d x)}^{q} \cong\left\|\mathbf{W}_{\alpha p, \frac{q}{q-p+1}}^{\infty} \mu\right\|_{L^{1}(d \mu)} \cong\left\|\mathbf{I}_{\alpha p} \mu\right\|_{L^{\frac{q}{p-1}}(d x)}^{\frac{q}{p-1}},
$$

where the constants in these equivalences are independent of $\mu$. 
We would like to acknowledge that Theorem 4.10 and 4.11 hold also in the general setting of homogeneous spaces (in the sense of Coifman and Weiss). In that context, the equivalence

$$
\left\|\mathbf{W}_{\alpha p, \frac{q}{q-p+1}}^{\infty} \mu\right\|_{L^{1}(d \mu)} \cong\left\|\mathbf{I}_{\alpha p} \mu\right\|_{L^{\frac{q}{p-1}}(d x)}^{\frac{q}{p-1}}
$$

was proved in $\mathrm{CO}$, and the equivalence

$$
\left\|\mathbf{W}_{\alpha, p}^{\infty} \mu\right\|_{L^{q}(d x)}^{q} \cong\left\|\mathbf{I}_{\alpha p} \mu\right\|_{L^{\frac{q}{p-1}(d x)}}^{\frac{q}{p-1}},
$$

along with its truncated and discrete counterparts, have been obtained recently in [HLX].

We are now in a position to prove the first main result of the paper.

Proof of Theorem 1.2. It is known that (i) $\Leftrightarrow$ (ii) at least in the elliptic case, i.e., on $\mathbb{R}^{N}$ (see, e.g., $\left.\mathrm{AH}\right]$ ), and the proof given in $[\mathrm{AH}]$ works also on Carnot groups. Next, by duality and Theorem 4.10 we have (i) $\Leftrightarrow$ (iii). Also, observe that (iii) $\Rightarrow$ (iv) by letting $g=\chi_{B}$ in (1.11). Moreover, we have the implication (iv) $\Rightarrow(\mathrm{v}$ ) by following the proof given in [PV1, Theorem 2.10] in the elliptic case. Thus from Theorem 4.3 we obtain the last conclusion of the theorem.

Therefore, it is left to show that the existence of a solution $u$ to (1.9) implies (i). To this end, we let $\mu=u^{q}+\omega$ and $\delta(x)=\operatorname{dist}(x, \partial \Omega)$. From the lower Wolff's potential estimate in Theorem 3.4 we have

$$
u(x) \geq C \mathbf{W}_{1, p}^{\frac{\delta(x)}{3}} \mu(x), \quad \forall x \in \Omega .
$$

By Lemma 4.8 we obtain for any $m \in \mathbb{Z}$,

$$
\left\{\sum_{\substack{Q \in \mathcal{D}_{m}, \ell(Q) \leq \lambda^{-3} \frac{\delta(x)}{3}}}\left[\frac{\mu(Q)}{|Q|^{1-\frac{p}{M}}}\right]^{\frac{1}{p-1}} \chi_{Q}(x)\right\}^{q} \chi_{\Omega}(x) d x \leq C d \mu,
$$

and thus

$$
\begin{aligned}
& \int_{\Omega}\left\{\sum_{\substack{Q \in \mathcal{D}_{m}, \ell(Q) \leq \lambda^{-3} \frac{\delta(x)}{3}}}\left[\frac{\mu(Q)}{|Q|^{1-\frac{p}{M}}}\right]^{\frac{1}{p-1}} \chi_{Q}(x)\right\}^{q}\left(\mathbf{M}_{\mu}^{\mathrm{dy}, \mathcal{D}_{m}} g\right)^{\frac{q}{p-1}} d x \\
& \leq C \int_{\mathbb{G}}\left(\mathbf{M}_{\mu}^{\mathrm{dy}, \mathcal{D}_{m}} g\right)^{\frac{q}{p-1}} d \mu,
\end{aligned}
$$

which holds for all $g \in L_{\mu}^{\frac{q}{p-1}}$. Here $\mathbf{M}_{\mu}^{\mathrm{dy}}, \mathcal{D}_{m}$ denotes the dyadic Hardy-Littlewood maximal function defined for a locally $\mu$-integrable function $f$ by

$$
\mathbf{M}_{\mu}^{\mathrm{dy}, \mathcal{D}_{m}} f(x)=\sup _{x \in Q \in \mathcal{D}_{m}} \frac{\int_{Q}|f| d \mu}{\mu(Q)} .
$$

Next, since $\mathbf{M}_{\mu}^{\mathrm{dy}}, \mathcal{D}_{m}$ is bounded on $L_{\mu}^{s}, s>1$, we get

$$
\int_{\Omega}\left\{\sum_{\substack{Q \in \mathcal{D}_{m}, \ell(Q) \leq \lambda^{-3} \frac{\delta(x)}{3}}}\left[\frac{\int_{Q} g d \mu}{|Q|^{1-\frac{p}{M}}}\right]^{\frac{1}{p-1}} \chi_{Q}(x)\right\}^{q} d x \leq C \int_{\mathbb{G}} g^{\frac{q}{p-1}} d \mu
$$


for all $g \in L_{\mu}^{\frac{q}{p-1}}, g \geq 0$. We now let $r_{0}=\operatorname{dist}(\operatorname{supp}(\omega), \partial \Omega)$ and $\Omega^{\prime}=\{x \in \Omega$ : $\left.\operatorname{dist}(x, \operatorname{supp}(\omega))<r_{0} / 2\right\}$, where the distance is taken with respect to the CarnotCarathéodory metric. Note that for $x \in \Omega$ with $\delta(x) \leq r_{0} / 4$, we have $Q \cap \Omega^{\prime}=\emptyset$ whenever $x \in Q \in \mathcal{D}_{m}$ and $\ell(Q) \leq \lambda^{-3} \frac{\delta(x)}{3}$. Inequality (4.19) then implies that for all $g \in L_{\mu}^{\frac{q}{p-1}}, g \geq 0$, we have

$$
\int_{\mathbb{G}}\left\{\sum_{\substack{Q \in \mathcal{D}_{m}, \ell(Q) \leq \lambda^{-3} \frac{r_{0}}{12}}}\left[\frac{\int_{Q} g d \mu}{|Q|^{1-\frac{p}{M}}}\right]^{\frac{1}{p-1}} \chi_{Q}(x)\right\}^{q} d x \leq C \int_{\mathbb{G}} g^{\frac{q}{p-1}} d \mu
$$

provided $\operatorname{supp}(g) \subset \Omega^{\prime}$. Thus for $k \in \mathbb{Z}$ such that $\lambda^{-4} \frac{r_{0}}{12} \leq \lambda^{k} \leq \lambda^{-3} \frac{r_{0}}{12}$, we find

$$
\sum_{j: E_{j}^{k} \in \mathcal{D}_{m}} \int_{E_{j}^{k}}\left\{\sum_{\substack{Q \in \mathcal{D}_{m} \\ Q \subset E_{j}^{k}}}\left[\frac{\int_{Q} g d \mu}{|Q|^{1-\frac{p}{M}}}\right]^{\frac{1}{p-1}} \chi_{Q}(x)\right\}^{q} d x \leq C \int_{\mathbb{G}} g^{\frac{q}{p-1}} d \mu,
$$

and hence by Proposition 4.6.

$$
\sum_{\substack{Q \in \mathcal{D}_{m}, \ell(Q) \leq \lambda^{-3} \frac{r_{0}}{12}}}\left[\frac{\int_{Q} g d \mu}{|Q|^{1-\frac{p}{M}}}\right]^{\frac{q}{p-1}}|Q| \leq C \int_{\mathbb{G}} g^{\frac{q}{p-1}} d \mu
$$

provided $\operatorname{supp}(g) \subset \Omega^{\prime}$. As (4.20) holds for all $m \in \mathbb{Z}$, it follows from Theorems 4.9 and 4.10 that

$$
\int_{\mathbb{G}}\left[\mathbf{G}_{p}(g d \mu)\right]^{\frac{q}{p-1}} d x \leq C \int_{\mathbb{G}} g^{\frac{q}{p-1}} d \mu
$$

for all $g \in L^{\frac{q}{p-1}}(d \mu), g \geq 0$, and $\operatorname{supp}(g) \subset \Omega^{\prime}$. Inequality (4.21), duality, and the facts that $\omega \leq \mu$ and $\operatorname{supp}(\omega) \subset \Omega^{\prime}$ finally yield

$$
\int_{\mathbb{G}} \mathbf{G}_{p}(f)^{\frac{q}{q-p+1}} d \omega \leq C \int_{\mathbb{G}} f^{\frac{q}{q-p+1}} d x
$$

for all $f \in L^{\frac{q}{q-p+1}}, f \geq 0$. This completes the proof of the theorem.

We next prove Theorem 1.3 .

Proof of Theorem 1.3. We first suppose that $C_{p, \frac{q}{q-p+1}}(E)=0$. Since $\frac{p q}{(q-p+1)}>p$ by Theorems 4.1 and 4.9 in $\left[\mathrm{Lu}\right.$ ] we find $C_{1, p}(E)=0$. On the other hand, by a result in [Fol] we have the identification

$$
S^{1, p}(\mathbb{G})=\mathbf{G}_{1}\left(L^{p}(\mathbb{G})\right)
$$

with $\|u\|_{S^{1, p}(\mathbb{G})} \cong\|f\|_{L^{p}(\mathbb{G})}$ for any $u$ with $u=\mathbf{G}_{1}(f)$. Thus we also have

$$
\operatorname{cap}_{1, p}(E, \Omega)=0
$$

where $\operatorname{cap}_{1, p}(\cdot, \Omega)$ is a relative capacity adapted to $\Omega$ (see [TW], [HKM]) defined by

$$
\operatorname{cap}_{1, p}(E, \Omega)=\inf \left\{\int_{\Omega}|X \varphi|^{p} d x: \varphi \in C_{0}^{\infty}(\Omega), \varphi \geq \chi_{E}\right\} .
$$


Let $u$ be a solution of (1.14). Using (4.22) and adapting the argument in HKM] to this setting, we see that the function

$$
\tilde{u}(x):=\left\{\begin{array}{c}
u(x), \quad x \in \Omega \backslash E, \\
\liminf _{y \rightarrow x, y \notin E} u(y), \quad x \in E
\end{array}\right.
$$

is a $p$-superharmonic extension of $u$ to the whole $\Omega$. We now let $\varphi$ be an arbitrary nonnegative function in $C_{0}^{\infty}(\Omega)$. As in $\mathrm{BP}$, Lemme 2.2], we can construct a sequence $\left\{\varphi_{n}\right\}$ of nonnegative functions in $C_{0}^{\infty}(\Omega \backslash E)$ such that

$$
0 \leq \varphi_{n} \leq \varphi ; \quad \varphi_{n} \rightarrow \varphi \quad \text { almost everywhere. }
$$

By Fatou's lemma we have

$$
\begin{aligned}
\int_{\Omega} \tilde{u}^{q} \varphi d x & \leq \liminf _{n \rightarrow \infty} \int_{\Omega} \tilde{u}^{q} \varphi_{n} d x=\liminf _{n \rightarrow \infty} \int_{\Omega} \varphi_{n} d \mu[\tilde{u}] \\
& \leq \int_{\Omega} \varphi d \mu[\tilde{u}]<+\infty .
\end{aligned}
$$

Here $\mu[\tilde{u}]$ is the measure generated by $\tilde{u}$. Therefore, $\tilde{u} \in L_{\mathrm{loc}}^{q}(\Omega)$, and $\mu[\tilde{u}] \geq \tilde{u}^{q}$ in $\mathcal{D}^{\prime}(\Omega)$. It is then easy to see that

$$
-\Delta_{\mathbb{G}, p} \tilde{u}=\tilde{u}^{q}+\mu \quad \text { in } \quad \mathcal{D}^{\prime}(\Omega)
$$

for some nonnegative measure $\mu$ supported on $E$. Moreover, by Theorem 1.2 we have

$$
\mu(E) \leq C(E) C_{p, \frac{q}{q-p+1}}(E)=0 .
$$

This gives $\mu=0$ and thus $\tilde{u}$ solves (1.14) with $\Omega$ in place of $\Omega \backslash E$.

Conversely, suppose that every solution to (1.14) can be extended to the whole $\Omega$. We will show $C_{p, \frac{q}{q-p+1}}(E)=0$ by a contradiction argument. To this end, suppose that $C_{p, \frac{q}{q-p+1}}(E)>0$ and consider the following equation

$$
\left\{\begin{aligned}
-\Delta_{\mathbb{G}, p} u & =u^{q}+\epsilon \mu^{E} \quad \text { in } \Omega \\
u & =0 \text { on } \partial \Omega
\end{aligned}\right.
$$

where $\mu^{E}$ is the capacitary measure of $E$ with respect to the capacity $C_{p, \frac{q}{q-p+1}}(\cdot)$ (see $[\mathrm{Lu}, \mathrm{AH}]$ ). Note that $\mu^{E}$ has the following important property (see, $[\mathrm{Lu}]$, AH] :

$$
\mathbf{G}_{p} *\left[\mathbf{G}_{p} * \mu^{E}\right]^{\frac{q-p+1}{p-1}} \leq 1 \quad \text { everywhere on } \operatorname{supp}(E) .
$$

Let $K$ be an arbitrary compact subset of $E$ and denote by $\mu_{K}$ the restriction of $\mu^{E}$ to $K$. We have

$$
\begin{aligned}
\int_{\mathbb{G}}\left[\mathbf{G}_{p} * \mu_{K}\right]^{\frac{q}{p-1}} d x & =\int_{K} \mathbf{G}_{p} *\left[\mathbf{G}_{p} * \mu_{K}\right]^{\frac{q-p+1}{p-1}} d \mu_{K} \\
& \leq \int_{K} \mathbf{G}_{p} *\left[\mathbf{G}_{p} * \mu^{E}\right]^{\frac{q-p+1}{p-1}} d \mu_{K} \\
& \leq \mu_{K}(K),
\end{aligned}
$$

where we used (4.26) in the last inequality. On the other hand, it follows from the dual definition of capacity (see (1.5)) that

$$
\mu_{K}(K) \leq C_{p, \frac{q}{q-p+1}}(K)^{\frac{q-p+1}{q}}\left\|\mathbf{G}_{p}\left(\mu_{K}\right)\right\|_{\frac{q}{p-1}(\mathbb{G})} .
$$


Thus we obtain from (4.27) and (4.28) that

$$
\mu^{E}(K)=\mu_{K}(K) \leq C_{p, \frac{q}{q-p+1}}(K) .
$$

Since this holds for all compact sets $K$, by Theorem 1.2 we see that the equation (4.25) is solvable as long as $\epsilon>0$ is chosen small enough. But this would give us a contradiction and hence the proof is complete.

\section{Global solutions and Liouville type theorems}

In this section we sketch the proofs of Theorem 1.4 and Corollary 1.5. To prove Theorem 1.4 one can proceed as in the proof of Theorem 1.2 but using Theorem 4.11 instead of Theorem 4.10, and the following global version of Theorem 4.3. The latter in turn can be proved as in [PV1, Theorem 5.3] by approximations and using pointwise estimates for potential theoretic solutions over arbitrarily large balls.

Theorem 5.1. Let $\omega \in \mathcal{M}^{+}(\mathbb{G}), 1<p<M$, and $q>p-1$. Suppose that

$$
\mathbf{W}_{1, p}^{\infty}\left[\left(\mathbf{W}_{1, p}^{\infty} \omega\right)^{q}\right] \leq C \mathbf{W}_{1, p}^{\infty} \omega<+\infty \quad \text { a.e. },
$$

where

$$
C \leq\left(\frac{q-p+1}{q A \max \left\{1,2^{p^{\prime}-2}\right\}}\right)^{q\left(p^{\prime}-1\right)}\left(\frac{p-1}{q-p+1}\right),
$$

and $A$ is the constant used in Definition 1.1. Then there exists a p-superharmonic function $u \in L_{\mathrm{loc}}^{q}\left(\mathbb{R}^{n}\right)$ such that

$$
\left\{\begin{aligned}
-\Delta_{\mathbb{G}, p} u & =u^{q}+\omega \\
\inf _{\mathbb{G}} u & =0
\end{aligned}\right.
$$

and for every $x \in \mathbb{G}$,

$$
c_{1} \mathbf{W}_{1, p}^{\infty} \omega(x) \leq u(x) \leq c_{2} \mathbf{W}_{1, p}^{\infty} \omega(x),
$$

where the constants $c_{1}, c_{2}$ depend only $p, q$, and $M$.

We remark that in order to show that the existence of a solution $u$ to (1.15) implies (i) and (ii) in Theorem 1.4, we need the following analogue of (4.21):

$$
\int_{\mathbb{G}}\left[\mathbf{I}_{p}(g d \mu)\right]^{\frac{q}{p-1}} d x \leq C \int_{\mathbb{G}} g^{\frac{q}{p-1}} d \mu
$$

where $\mu=u^{q}+\omega$. This can be shown to hold for all $g \in L^{\frac{q}{p-1}}(d \mu), g \geq 0$, with no restriction on the support of $g$ by using the lower bound in (3.3).

Finally, we give a proof of Corollary 1.5 .

Proof of Corollary 1.5. Corollary 1.5 follows from Theorem 1.4 and the fact that for $\alpha s \geq M$ the Riesz capacity $\dot{C}_{\alpha, s}(E)=0$ for every compact set $E \subset \mathbb{G}$. To see the latter, note that for any nonnegative measure $\mu$ supported in a ball $B_{R}(e)$, $R>0$, we have

$$
\mathbf{I}_{\alpha} * \mu(x)=\int_{B_{R}(e)} \frac{1}{d_{c c}(x, y)^{M-\alpha}} d \mu(y) \geq \frac{c \mu\left(B_{R}(e)\right)}{(|x|+R)^{M-\alpha}},
$$

where $|x|$ is the homogeneous norm of $x$ (see Section 2). Thus, using the condition $\alpha s \geq M$ and [Fol, Corollary 1.6], we get $\left\|\mathbf{I}_{\alpha} * \mu\right\|_{L^{\frac{s}{s-1}(\mathbb{G})}}=\infty$ unless $\mu$ is identically zero. Therefore, in view of the dual definition of capacity (see (1.6)) we obtain

$$
\dot{C}_{\alpha, s}(E)=0
$$

for every compact set $E \subset \mathbb{G}$. 


\section{REFERENCES}

[AH] D. R. Adams and L. I. Hedberg, Function Spaces and Potential Theory, Springer-Verlag, Berlin, 1996. MR1411441 (97j:46024)

[AP] D. R. Adams and M. Pierre, Capacitary strong type estimates in semilinear problems, Ann. Inst. Fourier (Grenoble), 41 (1991), 117-135. MR.1112194 (92m:35074)

[BP] P. Baras and M. Pierre, Critère d'existence des solutions positives pour des équations semi-linéaires non monotones, Ann. Inst. H. Poincaré, 3 (1985), 185-212. MR797270 (87j:45032)

[BV1] M. F. Bidaut-Véron, Necessary conditions of existence for an elliptic equation with source term and measure data involving p-Laplacian, Proc. 2001 Luminy Conf. on Quasilinear Elliptic and Parabolic Equations and Systems. Electron. J. Differ. Equ. Conf. 8 (2002), 23-34. MR1990293 (2004e:35055)

[BV2] M. F. Bidaut-Véron, Removable singularities and existence for a quasilinear equation with absorption or source term and measure data, Adv. Nonlinear Stud. 3 (2003), 25-63. MR:1955596(2003m:35093)

[BCC] I. Birindelli, I. Capuzzo Dolceltta and A. Cutri, Liouville theorems for semilinear equations on the Heisenberg group, Ann. Inst. H. Poincaré 14 (1997), 295-308. MR.1450950 (98d:35049)

[CDG] L. Capogna, D. Danielli, and N. Garofalo, An embedding theorem and the Harnack inequality for nonlinear subelliptic equations, Comm. Partial Differential Equations 18 (1993), 1765-1794. MR1239930 (94j:35038)

[CO] C. Cascante and J. Ortega, Norm inequalities for potential-type operators in homogeneous spaces, Math. Nachr. 228 (2001), 85-107. MR1845908(2002f:46047)

[COV] C. Cascante, J. M. Ortega, and I. E. Verbitsky, Nonlinear potentials and two weight trace inequalities for general dyadic and radial kernels, Indiana Univ. Math. J., 53 (2004), 845-882. MR.2086703 (2005g:42053)

[Cho] W. L. Chow, Uber systeme von linearen partiellen Differentialgleichungen erster Ordnung, Math. Annalen, 117 (1939), 98-105. MR0001880(1:313d)

[Chr] M. A. Christ, AT(b) theorem with remarks on analytic capacity and the Cauchy integral, Colloq. Math. 60/61 (1990), 601-628. MR1096400 (92k:42020)

[CLL] W. Cohn, G. Lu, and S. Lu, Higher order Poincaré inequalities associated with linear operators on stratified groups and applications Math. Z. 244 (2003), 309-335. MR.1992541 (2004d:46037)

[DMOP] G. Dal Maso, F. Murat, A. Orsina, and A. Prignet, Renormalized solutions of elliptic equations with general measure data, Ann. Scuol. Norm. Super. Pisa (4) 28 (1999), 741-808. MR 1760541(2001d:35190)

[Fol] G. B. Folland, Subelliptic estimates and function spaces on nilpotent Lie groups, Arkiv för Mat. 13 (1975), 161-207. MR0494315 (58:13215)

[GL] N. Garofalo and E. Lanconelli, Existence and nonexistence results for semilinear equations on the Heisenberg group, Indiana Univ. Math. J. 41 (1992), 71-97. MR.1160903 (93h:35071)

[HLX] X. Han, G. Lu, and Y. Xiao, Wolff potentials and regularity of solutions to integral systems on homogeneous spaces. Preprint 2011. MR2787437 (2012c:45007)

[HW] L. I. Hedberg and Th. H. Wolff, Thin sets in nonlinear potential theory, Ann. Inst. Fourier (Grenoble) 33 (1983), 161-187. MR727526 (85f:31015)

[HH] J. Heinonen and I. Holopainen, Quasiregular maps on Carnot groups, J. Geom. Anal. 7 (1997), 109-148. MR1630785 (99i:30037)

[HKM] J. Heinonen, T. Kilpeläinen, and O. Martio, Nonlinear Potential Theory of Degenerate Elliptic Equations, Oxford Univ. Press, Oxford, 1993. MR1207810 (94e:31003)

[Kil] T. Kilpeläinen, $p$-Laplacian type equations involving measures, Proceedings of the International Congress of Mathematicians, Vol. III (Beijing, 2002), 167-176. MR1957528 (2003j:35095)

[KM1] T. Kilpeläinen and J. Malý, Degenerate elliptic equations with measure data and nonlinear potentials, Ann. Scuola Norm. Super. Pisa, Cl. Sci. 19 (1992), 591-613. MR.1205885 (94c:35091)

[KM2] T. Kilpeläinen and J. Malý, The Wiener test and potential estimates for quasilinear elliptic equations, Acta Math. 172 (1994), 137-161. MR1264000 (95a:35050) 
[KR] A. Korányi and H. M. Reimann, Foundations for the theory of quasiconformal mappings on the Heisenberg group, Adv. Math. 111 (1995), 1-87. MR.1317384 (96c:30021)

[Lu] G. Lu, Potential analysis on Carnot groups, Part II: Relationship between Hausdorff measures and capacities, Acta Math. Sinica, English Series, 20 (2004), 25-46. MR2056553 (2005h:22013)

[NSW] A. Nagel, E. M. Stein, and S. Wainger, Balls and metrics defined by vector fields I: Basic properties, Acta Math. 155 (1985), 103-147. MR793239 (86k:46049)

[PV1] N. C. Phuc and I. E. Verbitsky, Quasilinear and Hessian equations of Lane-Emden type, Ann. Math. 168 (2008), 859-914. MR2456885(2010a:35075)

[PV2] N. C. Phuc and I. E. Verbitsky, Singular quasilinear and Hessian equations and inequalities, J. Funct. Analysis 256 (2009), 1875-1906. MR2498563 (2010f:35115)

[PVe] S. Pohozaev and L. Véron, Nonexistence results of solutions of semilinear differential inequalities on the the Heisenberg group, Manuscripta Math. 102 (2000), 85-99. MR1771229 (2001f:35451)

[Ra] P. K. Rashevsky, Any two points of a total nonholonomic space may be connected by an admissible line, Uch. Zap. Ped. Inst. Liebknecht, Ser. Phys. Math., (Russian) 2 (1938), 83-94.

[SW] E. T. Sawyer and R. L. Wheeden, Weighted inequalities for fractional integrals on Euclidean and homogeneous spaces, Amer. J. Math. 114 (1992), 813-874. MR.1175693 (94i:42024)

[Tu] B. O. Turesson, Nonlinear potential theory and weighted Sobolev spaces, Lecture Notes Math., 1736 (2000), 1-173. MR1774162 (2002f:31027)

[TW] N. S. Trudinger and X. J. Wang, On the weak continuity of elliptic operators and applications to potential theory, Amer. J. Math. 124 (2002), 369-410. MR.1890997 (2003c:35025)

[Va] V. S. Varadarajan, Lie Groups, Lie Algebras, and Their Representations, SpringerVerlag, New York-Berlin-Heidelberg-Tokyo, 1974. MR0376938 (51:13113)

[VSC] N. Th. Varopoulos, L. Saloff-Coste, and T. Coulhon, Analysis and Geometry on Groups, Cambridge Univ. Press, 1992. MR.1218884 (95f:43008)

Department of Mathematics, Louisiana State University, 303 Lockett Hall, Baton Rouge, Louisiana 70803

E-mail address: pcnguyen@math.1su.edu

Department of Mathematics, University of Missouri, Columbia, Missouri 65211

E-mail address: verbitskyi@missouri.edu 